\title{
ON LINEAR ALGEBRAIC SEMIGROUPS. II
}

BY

\author{
MOHAN S. PUTCHA
}

\begin{abstract}
We continue from [11] the study of linear algebraic semigroups. Let $S$ be a connected algebraic semigroup defined over an algebraically closed field $K$. Let $थ(S)$ be the partially ordered set of regular $g$-classes of $S$ and let $E(S)$ be the set of idempotents of $S$. The following theorems (among others) are proved. (1) $\mathcal{U}(S)$ is a finite lattice. (2) If $S$ is regular and the kernel of $S$ is a group, then the maximal semilattice image of $S$ is isomorphic to the center of $E(S)$. (3) If $S$ is a Clifford semigroup and $f \in E(S)$, then the set $\{e \mid e \in E(S), e>f\}$ is finite. (4) If $S$ is a Clifford semigroup, then there is a commutative connected closed Clifford subsemigroup $T$ of $S$ with zero such that $T$ intersects each $g$-class of $S$. (5) If $S$ is a Clifford semigroup with zero, then $S$ is commutative and is in fact embeddable in $\left(K^{n}, \cdot\right)$ for some $n \in Z^{+}$. (6) If $\mathrm{ch} \cdot K=0$ and $S$ is a commutative Clifford semigroup, then $S$ is isomorphic to a direct product of an abelian connected unipotent group and a closed connected subsemigroup of $\left(K^{n}, \cdot\right)$ for some $n \in Z^{+}$. (7) If $S$ is a regular semigroup and $\operatorname{dim} \cdot S<2$, then $|Q(S)|<4$. (8) If $S$ is a Clifford semigroup with zero and $\operatorname{dim} \cdot S=3$, then $|E(S)|=|\mathcal{Q}(S)|$ can be any even number > 8. (9) If $S$ is a Clifford semigroup then $\mathcal{U}(S)$ is a relatively complemented lattice and all maximal chains in $\mathcal{Q}(S)$ have the same number of elements.
\end{abstract}

1. Preliminaries. Let $S$ be an arbitrary semigroup. If $S$ has an identity element, then $S^{1}=S$. Otherwise $S^{1}=S \cup\{1\}, 1 \notin S$, with obvious multiplication. If $a, b \in S$, then $a \mid b$ ( $a$ divides $b$ ) if $b \in S^{1} a S^{1} . \mathcal{G}, \mathcal{R}, \mathcal{L}, \mathcal{H}, \mathscr{D}$ will denote the usual Green's relations on $S$. If $a \in S$, then we let $J_{a}, H_{a}$ denote the $\mathcal{G}$-class and $\mathcal{H}$-class of $a$ in $S$, respectively. If $a, b \in S$, then $J_{a} \leqslant J_{b}$ if $b \mid a . E(S)$ will denote the set of idempotents of $S$. If $e, f \in E(S)$, then $e \geqslant f$ if $e f=f e=f$. If $X \subseteq S$, then $C_{S}(X)=\{a \mid a \in S, a x=x a$ for all $x \in X\}$. We let $C(X)=C_{S}(X) \cap X$ and $E(X)=E(S) \cap X$. We let $\Omega(S)$ denote the maximal semilattice image of $S$. An ideal $I$ of $S$ is prime if for all $a, b \in S, a b \in P$ implies $a \in P$ or $b \in P$. If $a_{1}, \ldots, a_{n} \in S$, then $\left\langle a_{1}, \ldots, a_{n}\right\rangle$ denotes the subsemigroup of $S$ generated by $a_{1}, \ldots, a_{n}$. If $S$ has an identity element 1 , then we refer to the $\mathcal{H}$-class of 1 as the group of units of $S$. Let $a \in S$. Then $a$ is regular if $a \in a S a$. Let $I$ be an ideal of $S$. If $a \in I$, then $a$ is regular in $S$ if and only if $a$ is regular in $I$. Let $a, b \in I$ such that $a$ is regular. Then $a g b$ in $I$ if and only if $a g b$ is $S . S$ is regular if every element of $S$ is regular. $S$ is strongly $\pi$-regular if a power of each element of $S$ lies in a subgroup of $S$.

Let $S$ be a strongly $\pi$-regular semigroup, $a \in S$. Then it follows from Munn [10] that $a$ is regular if and only if $a g e$ for some $e \in E(S)$. In fact it is well known that $\mathscr{G}=\mathscr{D}$ for $S$. This fact is implicit in [10]. An explicit proof can be found in [6]. A

Received by the editors February 14, 1979 and, in revised form, August 29, 1979.

1980 Mathematics Subject Classification. Primary 20M10; Secondary 20 G99.

Key words and phrases. Linear algebraic semigroup, idempotent, subgroup, $f$-class.

(C) 1980 American Mathematical Society 0002-9947/80/0000-0258/\$06.25 
$g$-class $J$ of $S$ is regular if it contains an idempotent. Let $U(S)$ denote the set of all regular $\mathcal{G}$-classes of $S$. Then $\mathcal{Q}(S)$ becomes a partially ordered set with respect to the partial order on $g$-classes defined above.

Throughout this paper, $\mathbf{Z}, \mathbf{Z}^{+}, \mathbf{Q}^{+}, \mathbf{Q}, \mathbf{R}^{+}, \mathbf{R}$ will denote the sets of all integers, positive integers, positive rationals, rationals, positive reals and reals, respectively. If $X$ is a set then $\mathscr{P}(X)$ denotes the power set of $X$ and $|X|$ the cardinality of $X$. If $(P, \leqslant)$ is a partially ordered set and $\alpha, \beta \in P$, then $\alpha$ covers $\beta$ if $\alpha>\beta$ and there is no $\gamma \in P$ such that $\alpha>\gamma>\beta$. $K$ will denote a fixed algebraically closed field. If $X_{1}, \ldots, X_{n}$ are variables, then $K\left[X_{1}, \ldots, X_{n}\right]$ denotes the free commutative algebra over $K$ in variables $X_{1}, \ldots, X_{n}$ and $\mathscr{F}\left[X_{1}, \ldots, X_{n}\right]$ denotes the free commutative semigroup in variables $X_{1}, \ldots, X_{n}$. If $n \in \mathbf{Z}^{+}$then $K^{n}=K \times \cdots \times K$ is the affine $n$-space and $\Re_{n}(K)$ the algebra of all $n \times n$ matrices. $X \subseteq K^{n}$ is (Zariski) closed if it is the set of zeroes of a set of polynomials on $K^{n}$. If $X \subseteq K^{n}$ then $\bar{X}$ denotes the closure of $X$. Let $X \subseteq K^{m}, Y \subseteq K^{n}$ be closed, $\phi: X \rightarrow Y$. If $\phi=$ $\left(\phi_{1}, \ldots, \phi_{m}\right)$ where each $\phi_{i}$ is a polynomial, then $\phi$ is a morphism. $\phi$ is dominant if $\overline{\phi(X)}=Y$. By an algebraic semigroup we mean $(S, \circ)$ where $\circ$ is an associative operation on $S, S$ is a closed subset of $K^{n}$ for some $n \in \mathbf{Z}^{+}$and the map $(x, y) \rightarrow x \circ y$ is a morphism from $S \times S$ into $S$. Let $S, T$ be algebraic semigroups, $\phi: S \rightarrow T$ a (semigroup) homomorphism. Then $\phi$ is a ${ }^{*}$-homomorphism if $\phi$ is also a morphism (of varieties). If $\phi$ is a bijection and if both $\phi$ and $\phi^{-1}$ are *-homomorphisms, then we say that $\phi$ is a ${ }^{*}$-isomorphism and that $S, T$ are ${ }^{*}$-isomorphic. Let $S$ be an algebraic semigroup. Then by a result of W. E. Clark (see [11, Corollary 1.4]), there exists $n \in \mathbf{Z}^{+}$such that $a^{n}$ lies in a subgroup of $S$ for all $a \in S$. We let $\pi(S)$ denote the smallest such positive integer $n$. By the author [11, Corollary 1.11] there exist ideals $I_{0}, \ldots, I_{t}$ of $S$ such that $S=I_{t} \supseteq \cdots \supseteq I_{0}, I_{0}$ is the completely simple kernel of $S$ and each $I_{k} / I_{k-1}$ is either nil or completely 0-simple, $k=$ $1, \ldots, t$. In particular [11, Theorem 1.7], $\mathcal{Q}(S)$ is finite. Let $e, f \in E(S)$. Then $e g f$ if and only if $S e S=S f S$. Hence the set $\{S e S \mid e \in E(S)\}$ is also finite. Let $e \in E(S)$. Then $e S=\{a \mid a \in S, e a=a\}$ is closed. Similarly $e S e$ is closed. Since $e$ is the identity element of $e S e$, it follows [5, II, §2, Corollary 3.6] that $e S e \backslash H_{e}$ is closed. By a connected semigroup we mean an algebraic semigroup $S$ such that the underlying closed set is irreducible (i.e. is not a union of two proper closed subsets).

Let $S$ be an algebraic semigroup, $e \in E(S)$. If $G$ is the $\mathcal{H}$-class of $e$ then let $\tilde{G}=\{(a, b) \mid a, b \in S, a e=e a=a, b e=e b=b, a b=b a=e\}$. If $(a, b),(c, d) \in$ $\tilde{G}$, then define $(a, b)(c, d)=(a c, d b)$. $\tilde{G}$ then becomes an algebraic group isomorphic to $G$. If $(a, b) \in \tilde{G}$, then $(a, b)^{-1}=(b, a)$.

LEMma 1.1. Let $S$ be an algebraic semigroup, $e \in E(S), G=H_{e}$ Define $\theta: \tilde{G} \rightarrow S$ as $\theta(a, b)=a$. If $T$ is a closed subset of $\tilde{G}$ then $\overline{\theta(T)} \cap G=\theta(T)$. If $S$ is connected, then so is $\tilde{G}$ and $\operatorname{dim} \cdot \tilde{G}=\operatorname{dim} \cdot e S e$.

Proof. $e S e$ is a closed subsemigroup of $S$. If $S$ is connected, then by [11, Proposition 2.2], so is $e S e$. Hence we can assume without loss of generality that $e$ is the identity element of $S$. By [5, II, §2, Theorem 3.3] we can assume that $S$ is a closed submonoid of $\mathfrak{T}_{n}(K)$ for some $n \in \mathbf{Z}^{+}$. If $a \in S$, then let $f(a)$ denote the 
determinant of $a$. So $G=\{a \mid a \in S, f(a) \neq 0\}$. Let $G_{1}=\{(a, \alpha) \mid a \in G, \alpha \in K$, $f(a) \alpha=1\}$ with $(a, \alpha)(b, \beta)=(a b, \alpha \beta)$. Then $G_{1}$ is an algebraic group with $(a, \alpha)^{-1}=(\alpha \operatorname{adj} \cdot a, f(a))$. Define $\theta_{1}: G_{1} \rightarrow S$ as $\theta_{1}(a, \alpha)=a$. Let $T \subseteq G_{1}$ be closed. Suppose $T$ is the set of zeroes of $p_{1}, \ldots, p_{m} \in K\left[X_{1}, \ldots, X_{n^{2}}, Y\right]$ where $X_{1}, \ldots, Y$ are variables. So for $a \in G,(a, 1 / f(a)) \in T$ if and only if $p_{i}(a, 1 / f(a))$ $=0, i=1, \ldots, m$. Let $r$ denote the maximum of the degrees of $Y$ in $p_{1}, \ldots, p_{m}$. Let $q_{i}=p_{i}\left(X_{1}, \ldots, X_{n^{2}}, 1 / f\left(X_{1}, \ldots, X_{n^{2}}\right)\right)\left(f\left(X_{1}, \ldots, X_{n^{2}}\right)\right)^{r} \in K\left[X_{1}, \ldots, X_{n^{2}}\right]$. Then for $a \in G, p_{i}(a, 1 / f(a))=0$ if and only if $q_{i}(a)=0$. Hence if $T_{1}=\{a \mid a \in S$, $\left.q_{i}(a)=0, i=1, \ldots, m\right\}$, then $T_{1} \cap G=\theta(T)$. Since $T_{1}$ is closed, $\overline{\theta(T)} \subseteq T_{1}$ and $\overline{\theta(T)} \cap G=\theta(T)$. Next assume that $G_{1}$ is not connected. Then $G_{1}=A \cup B$ for some proper closed subsets $A, B$. Then $\theta_{1}(A) \neq G, \theta_{1}(B) \neq G$. So by above, $\overline{\theta_{1}(A)} \neq S, \overline{\theta_{1}(B)} \neq S$. Since $\theta_{1}\left(G_{1}\right)=G, S=\overline{\theta_{1}(A)} \cup \overline{\theta_{1}(B)} \cup(S \backslash G)$. Hence $S$ is not connected. Now define $\phi: G_{1} \rightarrow \tilde{G}$ as $\phi(a, \alpha)=(a, \alpha \operatorname{adj} \cdot a), \psi: \tilde{G} \rightarrow G_{1}$ as $\psi(a, b)=(a, f(b))$. Clearly $\phi=\psi^{-1}, \theta \circ \phi=\theta_{1}, \theta_{1} \circ \psi=\theta$. So $G_{1}$ and $\tilde{G}$ are *-isomorphic and the lemma is proved.

LEMMA 1.2. Let $S$ be an algebraic semigroup, $J \in \mathcal{Q}(S)$. Let $G_{1}, G_{2}$ be two maximal subgroups of $J$. Then $\tilde{G}_{1}$ is *-isomorphic to $\tilde{G}_{2}$.

Proof. By the Rees-Green theorems (see [3, Chapter 2]) there exist $a, b, c, d \in J$ such that the maps $\phi: G_{1} \rightarrow G_{2}, \psi: G_{2} \rightarrow G_{1}$ given by $\phi(x)=a x b, \psi(y)=c y d$ are isomorphisms and $\psi=\phi^{-1}$. Define $\phi_{1}: \tilde{G}_{1} \rightarrow \tilde{G}_{2}, \psi_{1}: \tilde{G}_{2} \rightarrow \tilde{G}_{1}$ as $\phi_{1}\left(x, x^{-1}\right)=(\phi(x)$, $\left.\phi\left(x^{-1}\right)\right), \psi_{1}\left(y, y^{-1}\right)=\left(\psi(y), \psi\left(y^{-1}\right)\right)$. Then $\phi_{1}^{-1}=\psi_{1}$ and $\phi_{1}$ is a ${ }^{*}$-isomorphism.

LemMA 1.3. Let $S$ be a semigroup, $e, f \in E(S), e \mid f$. Then there exists $f^{\prime} \in E(S)$ such that $f g f^{\prime}$ and $e \geqslant f^{\prime}$.

Proof. $x e y=f$ for some $x, y \in S$. Let $f^{\prime}=$ eyfxe.

Corollary 1.4. Let $S$ be a semigroup. Suppose $S$ has a kernel $M$ which is a group. Let $f \in E(M)$. Then $e \geqslant f$ for all $e \in E(S)$.

Proof. Let $e \in E(S)$. Then $e \mid f$. By Lemma $1.3, e \geqslant f$.

LEMMA 1.5. Let $S$ be a strongly $\pi$-regular semigroup with identity element 1 . Then the nonunits of $S$, if nonempty, form a prime ideal of $S$.

Proof. Let $a, b \in S$ such that $a b=1$. Then $a^{m} b^{m}=1$ for all $m \in \mathbf{Z}^{+}$. There exists $n \in \mathrm{Z}^{+}$such that $a^{n} \mathcal{H} e$ for some $e \in E(S)$. Then $e a^{n}=a^{n}$ and so $e=e \cdot 1$ $=e a^{n} b^{n}=a^{n} b^{n}=1$. Hence $a^{n} \mathcal{H} 1$. It follows that $a \mathcal{H} 1$. Now let $G$ be the $\mathcal{H}$ class of 1 and let $P=S \backslash G$. Suppose $P \neq \varnothing$. Let $a \in P, x \in S$. Suppose $a x \in G$. Then $a b=1$ for some $b \in S$ and $a \in G$ by the above, a contradiction. Hence $a x \in P$. It follows that $P$ is right ideal of $S$. Similarly $P$ is a left ideal of $S$. This proves the lemma.

LEMMA 1.6. Let $S$ be a strongly $\pi$-regular semigroup such that $\mathscr{U}(S)$ is finite. Suppose $S$ is a semilattice of semigroups $S_{\alpha}(\alpha \in \Gamma)$. Then:

(1) Each $S_{\alpha}(\alpha \in \Gamma)$ is strongly $\pi$-regular.

(2) $थ(S)=\cup_{\alpha \in \Gamma}$ थ $\left(S_{\alpha}\right)$.

(3) Each $S_{\alpha}(\alpha \in \Gamma)$ has a completely simple kernel. 
Proof. Let $\alpha \in \Gamma, a \in S_{\alpha}$. Then $a^{n}$ lies in a subgroup $G$ of $S$. Since $G \cap S_{\alpha} \neq$ $\varnothing, G \subseteq S_{\alpha}$. Hence $S_{\alpha}$ is strongly $\pi$-regular. Next let $e \in E\left(S_{\alpha}\right), a \in S_{\alpha}$ such that $e g_{a}$ in $S$. Then there exist $x, y, s, t \in S^{1}$ such that $x e y=a$, sat $=e$. Let $x^{\prime}=x e$, $y^{\prime}=e y, s^{\prime}=e s, t^{\prime}=t e$. Then $x^{\prime}, y^{\prime}, s^{\prime}, t^{\prime} \in S_{\alpha}, x^{\prime} e y^{\prime}=a, s^{\prime} a t^{\prime}=e$. So $a g e$ in $S_{\alpha}$. Hence $\mathscr{U}(S)=\cup_{\alpha \in \Gamma} \mathcal{U}\left(S_{\alpha}\right)$. In particular, $\mathscr{U}\left(S_{\alpha}\right)$ is finite. By [11, Lemma 1.9], each $S_{\alpha}$ has a completely simple kernel.

LeMma 1.7. Let $S$ be a semigroup, $e \in E(S)$ and set $T=e S e$. Then for $a, b \in T$, $a \mid b$ in $T$ if and only if $a \mid b$ in $S$.

Proof. Let $a, b \in T$ such that $a \mid b$ in $S$. Then $x a y=b$ for some $x, y \in S^{1}$. So $($ exe $) a(e y e)=$ exaye $=e b e=b$. So $a \mid b$ in $T$.

LEMMA 1.8. Let $n \in \mathbf{Z}^{+}, n \geqslant 3$. Then there exist $u_{1}, \ldots, u_{n}$ in the free commutative semigroup $\mathscr{F}\left(X_{1}, X_{2}, X_{3}\right)$ such that $u_{i}^{t} \notin\left\langle u_{1}, \ldots, u_{i-1}, u_{i+1}, \ldots, u_{n}\right\rangle$ for any $t \in \mathbf{Z}^{+}, i \in\{1, \ldots, n\}$.

Proof. Let $a_{1}, \ldots, a_{n}$ be the vertices of a regular $n$-gon in $\mathbf{R}^{+} \times \mathbf{R}^{+}$. Then no $a_{i}$ is a convex linear combination of the remaining $a_{j}$ 's. If we choose $b_{1}, \ldots, b_{n} \in \mathbf{Q}^{+}$ $\times \mathbf{Q}^{+}$close enough to $a_{1}, \ldots, a_{n}$ then no $b_{i}$ is a convex linear combination of the remaining $b_{j}$ 's. If $m \in Z^{+}$then $m b_{1}, \ldots, m b_{n}$ have the same property. So we can assume without loss of generality, that $b_{1}, \ldots, b_{n} \in \mathbf{Z}^{+} \times \mathbf{Z}^{+}$. Let $b_{i}=\left(\alpha_{i}, \beta_{i}\right)$, $i=1, \ldots, n$. Set $u_{i}=X_{1}^{\alpha_{i}} X_{2}^{\beta_{i}} X_{3}$. Suppose $u_{i}^{t} \in\left\langle u_{1}, \ldots, u_{i-1}, u_{i+1}, \ldots, u_{n}\right\rangle$ for some $t \in \mathbf{Z}^{+}, i \in\{1, \ldots, n\}$. Relabeling the $u_{j}$ 's if necessary, we can assume that $i=1$. So $u_{1}^{t}=u_{2}^{t_{2}} \cdots u_{n}^{t_{n}}$ for some $t_{2}, \ldots, t_{n} \in \mathbf{Z}, t_{2}, \ldots, t_{n}>0$. Then $X_{3}^{t}=$ $X_{3}^{t_{2}} \cdots X_{3}^{t_{n}}$. so $t_{2}+\cdots+t_{n}=t$ and $t_{2} b_{2}+\cdots+t_{n} b_{n}=t b_{1}$. So $r_{2} b_{2}$ $+\cdots+r_{n} b_{n}=b_{1}$, where $r_{j}=t_{j} / t, j=2, \ldots, n$. So $b_{1}$ is convex linear combination of $b_{2}, \ldots, b_{n}$, a contradiction. This proves the lemma.

LEMMA 1.9. Let $S$ be an algebraic semigroup with identity element 1 . Then 1 lies in a unique irreducible component $T$ of $S$. Moreover $T$ is a subsemigroup of $S$.

Proof. Let $T_{1}, T_{2}$ be irreducible components of $S$ such that $1 \in T_{1} \cap T_{2}$. Let $\phi$ : $T_{1} \times T_{2} \rightarrow S$ be given by $\phi(a, b)=a b$. So $\overline{\phi\left(T_{1} \times T_{2}\right)}$ is irreducible and $T_{i} \subseteq$ $\phi\left(T_{1} \times T_{2}\right)=T_{1} T_{2}, i=1,2$. Hence $T_{1}=T_{2}=T_{1} T_{2}$.

LemMa 1.10. Let $S$ be a connected semigroup, $e \in E(S)$. Then the following conditions are equivalent.

(1) $e \in C(S)$,

(2) $e \in C(E(S))$,

(3) $e S=S e$,

(4) $J_{e}$ is a group.

Proof. Suppose $e S=S e$. Then for any $x \in S$, ex $\in S e$ and hence exe $=e x$. Similarly $e x e=x e$. So $e x=x e$ and $e \in C(S)$. Hence $(3) \Rightarrow(1)$. That $(1) \Rightarrow(2)$ and (3) is obvious. That (4) $\Rightarrow(1)$ and $(2) \Rightarrow(1)$ follows from [11, Theorem 2.7]. That $(1) \Rightarrow(4)$ follows from [11, Lemma 2.9]. 
LeMmA 1.11. Let $\Gamma, \Lambda$ be finite sets, $\phi: \Gamma \rightarrow \mathcal{P}(\Lambda), \psi: \Lambda \rightarrow \mathscr{P}(\Gamma)$, such that for all $\alpha \in \Gamma, \beta \in \Lambda,|\phi(\alpha)|=|\psi(\beta)|=2$ and $\beta \in \phi(\alpha)$ if and only if $\alpha \in \psi(\beta)$. Then $|\Gamma|=|\Lambda|$.

Proof. We prove by induction on $|\Gamma|$. If $\alpha \in \Gamma$ and $\beta \in \Lambda$, then define $\alpha-\beta$ and $\beta-\alpha$ if $\beta \in \phi(\alpha)$. Let $\alpha_{1} \in \Gamma$. Then $\alpha_{1}-\beta_{1}$ for some $\beta_{1} \in \Lambda$. Choose $m \in \mathbf{Z}^{+}$maximal such that there exist distinct $\alpha_{1}, \ldots, \alpha_{m} \in \Gamma$, distinct $\beta_{1}, \ldots, \beta_{m}$ $\in \Lambda$ such that

$$
\alpha_{1}-\beta_{1}-\alpha_{2}-\cdots-\alpha_{m}-\beta_{m} .
$$

Since $\left|\psi\left(\beta_{m}\right)\right|=2$, there exists $\alpha \in \Gamma, \alpha \neq \alpha_{m}$ such that $\beta_{m}-\alpha$. Suppose $\alpha \notin$ $\left\{\alpha_{1}, \ldots, \alpha_{m}\right\}$. Since $|\phi(\alpha)|=2$, there exists $\beta \in \Lambda$ such that $\alpha-\beta$ and $\beta \neq \beta_{m}$. Since $\psi\left(\beta_{i}\right)=\left\{\alpha_{i}, \alpha_{i+1}\right\}$ for $i<m$, we see that $\beta \notin\left\{\beta_{1}, \ldots, \beta_{m}\right\}$. This contradicts the maximality of $m$. Hence $\alpha \in\left\{\alpha_{1}, \ldots, \alpha_{m}\right\}$ and $m>2$. So $\alpha=\alpha_{i}$ for some $i<m$. Suppose $i>1$. Then $\phi\left(\alpha_{i}\right)=\left\{\beta_{i-1}, \beta_{i}, \beta_{m}\right\}$ a contradiction. Hence $i=1$ and $\beta_{m}-\alpha_{1}$. Set $\alpha_{m+1}=\alpha_{1}, \beta_{0}=\beta_{m}$. Then $\phi\left(\alpha_{i}\right)=\left\{\beta_{i-1}, \beta_{i}\right\}, \psi\left(\beta_{i}\right)=$ $\left\{\alpha_{i}, \alpha_{i+1}\right\}, i=1, \ldots, m$. Let $\Gamma_{1}=\Gamma \backslash\left\{\alpha_{1}, \ldots, \alpha_{m}\right\}, \Lambda_{1}=\Lambda \backslash\left\{\beta_{1}, \ldots, \beta_{m}\right\}$. Then $\phi\left(\Gamma_{1}\right) \subseteq \mathscr{P}\left(\Lambda_{1}\right), \psi\left(\Lambda_{1}\right) \subseteq \mathscr{P}\left(\Gamma_{1}\right)$. By our induction hypothesis, $\left|\Gamma_{1}\right|=\left|\Lambda_{1}\right|$. Thus $|\Gamma|=|\Lambda|$, proving the lemma.

\section{Ideals and regular $\mathcal{G}$-classes.}

LEMMA 2.1. Let $S$ be an algebraic semigroup, $e \in E(S)$. Then $I=\{a \mid a \in S, a \nmid e\}$ is closed.

Proof. $e S e \backslash H_{e}$ is closed and hence $T=\left\{a \mid\right.$ exaye $\in e S e \backslash H_{e}$ for all $\left.x, y \in S\right\}$ is closed. Let $a \in S, a \notin I$. Then $a \mid e$. So $x a y=e$ for some $x, y \in S$. Hence exaye $\in H_{e}$ and $a \notin T$. Next assume $a \in S, a \notin T$. Then exaye $\in H_{e}$ for some $x, y \in S$. So a|exaye $\mid e$. Then $a \mid e$ and $a \notin I$. Hence $I=T$ is closed.

LEMma 2.2. Let $S$ be an algebraic semigroup, $\pi(S)=n$. Let $e \in E(S)$. Then $M=\left\{a \mid a \in S, a^{n} \in S e S\right\}$ is closed.

Proof. Let $E=E(S)$. If $f \in E$, then let $B_{f}=\{a \mid a \in S, a \nmid f\}$. Then $B_{f}$ is closed by Lemma 2.1. Let $F=\{f \mid f \in E$, ełf $\}$. Then $S e S \subseteq B_{f}$ for all $f \in F$. So $\operatorname{Se} S \subseteq B=\bigcap_{f \in F} B_{f}$ and $B$ is closed. Let $a \in B$. Now $a^{n} \mathcal{Y} f$ for some $f \in E$. So $a \notin B_{f}, f \notin F$. So $e|f| a^{n}$ and $a^{n} \in$ SeS. Hence $a^{n} \in$ SeS for all $a \in B$. Let $M_{1}=\left\{a \mid a \in S, a^{n} \in B\right\}$. Since $B$ is closed, so is $M_{1}$. Since $S e S \subseteq B, M \subseteq M_{1}$. Let $a \in M_{1}$. Then $a^{n} \in B$. Hence $a^{n^{2}} \in \operatorname{SeS}$. Since $s^{n} \mathcal{H} a^{n^{2}}, a^{n} \in \operatorname{SeS}$. Thus $a \in M$ and $M=M_{1}$ is closed.

THEOREM 2.3. Let $S$ be an algebraic semigroup, $\pi(S)=n, I$ an ideal of $S$. Then

(1) $M=\left\{a \mid a \in S, a^{n} \in I\right\}$ is closed.

(2) $\bar{I}$ is an ideal of $S, \bar{I} / I$ is nil and for all $a \in \bar{I}, a^{n} \in I$.

Proof. Let $E=E(S), F=E \cap I$. If $f \in F$, then let $M(f)=\left\{a \mid a \in S, a^{n} \in\right.$ $S f S$ \}. By Lemma 2.2, $M(f)$ is closed for all $f \in F$. Let $M_{1}=\cup_{f \in F} M(f)$. Since $\mathcal{Q}(S)$ is finite, the family $\{M(f) \mid f \in F\}$ is finite. Hence $M_{1}$ is closed. Clearly $M_{1} \subseteq M$. Let $a \in M$. Then $a^{n} \in I$. Now $a^{n} \mathcal{H} f$ for some $f \in E$. Since $a^{n} \in I$, 
$f \in F$. Hence $a^{n} \in S f S$ and $a \in M(f) \subseteq M_{1}$. Thus $M=M_{1}$ is closed. Since $I \subseteq M, \bar{I} \subseteq M$. It suffices to show that $\bar{I}$ is an ideal of $S$. Let $I_{1}=\{a \mid a \in S$, $x a y \in \bar{I}$ for all $\left.x, y \in S^{1}\right\}$. Then $I_{1}$ is closed and $I \subseteq I_{1}$. Hence $\bar{I} \subseteq I_{1}$ and $\bar{I}$ is an ideal of $S$. This proves the theorem.

COROLlaRY 2.4. Let $S$ be a regular algebraic semigroup. Then $S$ has only finitely many ideals and every ideal of $S$ is closed.

Proof. Let $I$ be an ideal of $S$. By Theorem $2.3, \bar{I}$ is an ideal of $S$ and $\bar{I} / I$ is nil. Since $S$ is regular, so are $\bar{I}$ and $\bar{I} / I$. It follows that $\bar{I}=I$. Since $\mathcal{U}(S)$ is finite, $S$ has only finitely many $q$-classes. Since every ideal of $S$ is a union of the $G$-classes contained in it, $S$ has only finitely many ideals.

By [11, Proposition 2.2] and Corollary 2.4 we have

Corollary 2.5. Let $S$ be a connected regular semigroup, $e \in E(S)$. Then eS, Se, $e S e$ and $\mathrm{SeS}$ are closed connected subsemigroups of $S$.

THEOREM 2.6. Let $S$ be a connected semigroup. Then the following conditions are equivalent.

(1) Every ideal of an ideal of $S$ is closed.

(2) $S$ is regular.

Proof. First assume $S$ is regular. Then clearly every ideal of an ideal of $S$ is an ideal of $S$. By Corollary 2.4, every ideal of $S$ is closed. Assume conversely that every ideal of an ideal of $S$ is closed. Suppose $S$ is not regular. Choose a nonregular connected ideal $T$ of $S$ of smallest possible dimension. Then every ideal of $T$ is closed. We claim $T^{2}=T$. For suppose $T^{2} \neq T$. Let $x \in T \backslash T^{2}$. Then $T \backslash\{x\}$ is an ideal of $T$ and hence closed. Since $T=(T \backslash\{x\}) \cup\{x\}$ and $T$ is connected, we see that $T=\{x\}$ and $x^{2}=x$, a contradiction. Hence $T^{2}=T$. Let $A=\{a \mid a \in T$, $a$ is not regular $\}$. We claim that there exists $a \in A$ such that $T a T=T$. Suppose not. Then $T a T \neq T$ for all $a \in A$. Then $T a T$ is an ideal of $S$ and hence closed. Since $T a T$ is the closed image of irreducible set $T \times T$ under the morphism $(x, y) \rightarrow x a y, T a t$ is also connected. Since $T a T \subset T, \operatorname{dim} \cdot T a T<\operatorname{dim} \cdot T$. By our induction hypothesis, $T a T$ is regular. In particular, $a \notin T a T$ for all $a \in A$. Again let $a \in A$. Then since $T=T^{2}$, there exist $b, c \in T$ such that $b c=a$. If $b, c$ are both regular, then clearly $a \in T a T$, a contradiction. By symmetry we may assume that $b \in A$. Hence $T b T$ is a closed connected regular subsemigroup of $T$. Let $M=S^{1} b T$. Then $M$ is an ideal of $S$ and so every ideal of $M$ is closed. Let $\mathscr{K}=\{X \mid T b T \subseteq X \subseteq M\}$. Let $X \in \mathcal{K}$. Since $M^{2} \subseteq T b T, X$ is an ideal of $M$. Hence $X$ is closed for all $X \in \mathscr{K}$. If $\mathscr{K}$ is infinite, we obtain a contradiction to the fact that closed sets satisfy $D \cdot C \cdot C$ (Hilbert Basis Theorem). Hence $\mathscr{K}$ is finite and $M \backslash T b T$ is finite. Let $W=\overline{b T}$. Since $b T \subseteq M, W \subseteq M$. Define $\phi: T \rightarrow M$ as $\phi(x)=b x$. Since $T$ is irreducible, so is $W=\overline{\phi(T)}$. Since $T b T$ is regular, $a \notin T b T$. Since $a \in b T \subseteq W, W \nsubseteq T b T$. Now $W \subseteq M=(M \backslash T b T) \cup T b T$. Since $W$ is irreducible and $M \backslash T b T$ is finite, it follows that $|W|=1$. Hence $|b T|=1$ and $b T=\{a\}$. So $a^{2}=a$, a contradiction. This contradiction shows that there exists $a \in A$ such that $T a T=T$. Let $I=\cup_{e \in E(T)} T e T$. Then $I$ is an ideal of $T$ and $T / I$ 
is nil. Since $T^{2}=T$, there exist $x, y \in T$ such that $x y=a$. Since $T a T=T$, there exist $u, v \in T$ such that $y=u a v$. So $x u a v=a$ and $(x u)^{r} a v^{r}=a$ for all $r \in \mathbf{Z}^{+}$. But $v^{t} \in I$ for some $t \in \mathbf{Z}^{+}$. Hence $a \in I$. So $a \in T e T$ for some $e \in E(T)$. Since $T a T=T, a g e$ and $a$ is regular. This contradiction proves the theorem.

In answer to a problem of Rhodes [12], Hall [7] (and independently, C. J. Ash) showed that every finite partially ordered set with a minimum element occurs as the partially ordered set of $\mathscr{f}$-classes of a finite inverse semigroup. We show that the situation is quite different for connected semigroups.

THEOREM 2.7. Let $S$ be a connected semigroup. Then $\mathcal{U}(S)$ is a finite lattice.

Proof. Let $\pi(S)=n, E=E(S)$ and let $T$ denote the kernel of $S$. If $e \in E$ then let $M(e)=\left\{a \mid a^{n} \in S e S\right\}$. By Lemma 2.2, M(e) is closed. Since $\mathcal{Q}(S)$ is finite, $\mathscr{B}=\{M(e) \mid e \in E\}$ is finite. Let $e, f \in E$. We will show that $J_{e} \wedge J_{f}$ exists in $\mathcal{Q}(S)$. Let $A=\{g|g \in E, e| g, f \mid g\}$. Since $E(T) \subseteq A, A \neq 0$. Let $I=$ $\cup_{g \in A} S g S, I_{1}=\cup_{g \in A} M(g)$. Then $I \subseteq I_{1}$. Let $x, y, z \in S$. Then $(x e y f z)^{n} \mathcal{H} g$ for some $g \in E$. Clearly $e \mid g$ and $f \mid g$. So $g \in A$. Thus xeyfz $\in M(g) \subseteq I_{1}$. Define $\phi$ : $S \times S \times S \rightarrow I_{1}$ as $\phi(x, y, z)=x e y f z$. Let $a \in I$. Then $a \in S g S$ for some $g \in A$. So $g \in S e S \cap S f S$. Hence $a \in S g S=S g^{2} S \subseteq S S e S S f S S \subseteq S e S . f S=\phi(S \times S \times$ $S)$. So $I \subseteq \phi(S \times S \times S)$. Since $S \times S \times S$ is irreducible, $\mathscr{B}$ is finite and $\phi(S \times S \times S) \subseteq I_{1}=\cup_{g \in A} M(g)$, we see that $\phi(S \times S \times S) \subseteq M(h)$ for some $h \in A$. Hence $I \subseteq M(h)$. Thus $h \mid g$ for all $g \in A$ and $J_{e} \wedge J_{f}=J_{h}$. $Q(S)$ is therefore a $\wedge$-semilattice. To show that $\mathcal{Q}(S)$ is a lattice, it suffices to show (since $\mathcal{U}(S)$ is finite) that $\mathscr{U}(S)$ has a maximum element. Clearly $S=\cup_{e \in E} M(e)$. Since $\mathscr{B}$ is finite and $S$ is connected, $S=M(f)$ for some $f \in E$. So $e \in S f S$ for all $e \in E$ and $J_{f}$ is maximum in $\mathscr{U}(S)$. This proves the theorem.

LEMMA 2.8. Let $S$ be a connected regular semigroup, $f \in E(S), S f S=S$, $\operatorname{dim} \cdot S$ $=n, \operatorname{dim} \cdot f S=m$. Let $A=\{x \mid x \in S, f x=f\}$. Then $\operatorname{dim} \cdot A=n-m$.

Proof. Let $Y=f S$, and let $\phi: S \rightarrow Y$ be given by $\phi(x)=f x$. Then $\phi$ is surjective. Let $I=S \backslash J_{f}$. Then $I$ is an ideal of $S$ and is hence closed. Let $W=J_{f} \cap Y, F=I \cap Y$. Let $a \in W, B=\phi^{-1}(a), A=\phi^{-1}(f)$. So $f a=a$. Since $a q f, a \Re f$. So there exists $u \in S$ such that $a u=f$. If $x \in A$, then let $\mu(x)=x a$. Then $f \mu(x)=f x a=f a=a$. Hence $\mu: A \rightarrow B$. If $y \in B$, then let $\nu(y)=y u$. So $f \nu(y)=f y u=a u=f$. Hence $\nu: B \rightarrow A$. Let $x \in A$. Then $f x=f$. Since $S f S=S$,

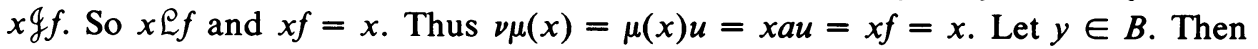
$f y=a$. Since $S f S=S$ and $a \mathscr{f} f, y g a$. So $y \_a$ and $w a=y$ for some $w \in S$. Hence $\mu \nu(y)=\nu(y) a=y u a=w a u a=w f a=w a=y$. Thus $\mu=\nu^{-1}$ and $A, B$ are isomorphic as varieties. Thus $\operatorname{dim} \cdot \phi^{-1}(a)=\operatorname{dim} \cdot \phi^{-1}(f)$ for all $a \in W$. Suppose $\operatorname{dim} \cdot \phi^{-1}(f)>n-m$. The $\operatorname{dim} \cdot \phi^{-1}(a)>n-m$ for all $a \in W$. By [13, Chapter I, $\S 6$, Theorem 7] there exists a nonempty open set $U$ of $Y$ such that $\operatorname{dim} \cdot \phi^{-1}(b)=n$ - $m$ for all $b \in U$. By the above, $U \subseteq F$. Thus $Y=F \cup(Y \backslash U)$ and $F, Y \backslash U$ are proper closed subsets of $Y$. This contradicts the irreducibility of $Y$. Hence $\operatorname{dim} \cdot \phi^{-1}(f)=n-m$. 
LEMma 2.9. Let $S$ be a connected regular semigroup, e, $f \in E(S), e \mid f$. Then there exists $e_{1} \in E(S)$ such that $e g e_{1}$ and $f e_{1}=f$.

Proof. If $e f f$, then there is nothing to prove. So assume $e f f$. Since $\mathcal{U}(S)$ is finite, we are easily reduced to the case when $J_{e}$ covers $J_{f}$ in $Q(S)$. We can also assume that $S e S=S$ (otherwise we work with $S e S$ ). Let $T=S f S, n=\operatorname{dim} \cdot S$, $m=\operatorname{dim} \cdot T, p=\operatorname{dim} \cdot f S$. Then $T f T=T$ and $p \leqslant m<n$. Define $\phi: S \rightarrow f S$ as $\phi(x)=f x$. Let $A=\phi^{-1}(f)=\{x \mid x \in S, f x=f\}$. Suppose $A \subseteq T$. Then by Lemma 2.8, $\operatorname{dim} \cdot A=m-p<n-p$, contradicting [9, Theorem 4.1]. Hence $A \nsubseteq T$. So there exists $x \in S, x \notin T$ such that $f x=f$. Since $S e S=S, e|x| f$. Since $J_{e}$ covers $J_{f}, e \mathcal{G}$. Now $x \Re e_{1}$ for some $e_{1} \in E(S)$. Hence $f e_{1}=f, e_{1} g e$. This proves the lemma.

THEOREM 2.10. Let $S$ be a connected regular semigroup such that the kernel of $S$ is a group. Suppose $J \in \mathcal{Q}(S), e \in E(J), J^{2} \subseteq J$. Then $e \in C(S)$ and $J$ is a group.

Proof. By Lemma 1.10, it suffices to show that $e S=S e$. Since $e(S e S)=e S$ and $(\mathrm{SeS}) e=S e$, we can assume, without loss of generality, that $S=S e S$. By symmetry it suffices to show that $S e=S$. Let $M$ denote the kernel of $S$ and let $E(M)=\{g\}$. By Corollary 1.4, $e \geqslant g$. Suppose $S e \neq S$. Define $\phi: S \rightarrow S e$ as $\phi(x)=x e$. Clearly $g \in S e$. Since $\operatorname{dim} \cdot S e<\operatorname{dim} \cdot S$, we see [9, Theorem 4.1] that $\phi^{-1}(g) \neq\{g\}$. So there exists $x \in S, x \neq g$ such that $x e=g$. Now $x \mathscr{l} f$ for some $f \in E(S)$. Since $e \mid f$, we see by Lemma 2.9 that there exists $e_{1} \in E(S)$ such that $e g e_{1}$ and $f e_{1}=f$. So $x e_{1}=x$. Since $J^{2} \subseteq J, e_{1} e g e_{1}$. Hence $e_{1} e \Re e_{1}$ and there exists $y \in S$ such that $e_{1} e y=e_{1}$. Hence $x=x e_{1}=x e_{1} e y=x e y=g y \in M$. So $x \in M$ and $x g=x$. Since $x e=g$ and $e \geqslant g$ we see that $x=x g=x e g=g$, a contradiction. This proves the theorem.

THEOREM 2.11. Let $S$ be a connected regular semigroup such that the kernel of $S$ is a group. Then $\Lambda=E(C(S))=C(E(S)) \neq \varnothing$. If $a \in S$, then let $N(a)=\{e \mid e \in \Lambda$, ae $\mathcal{H}$ e $\}$. Define $\delta$ on $S$ as $a \delta b$ if and only if $N(a)=N(b)$. Then $\delta$ is the finest semilattice congruence on $S$. In particular, $\Omega(S) \cong \Lambda$.

Proof. By Lemma 1.10, $C(E(S))=E(C(S))$. Let $M$ be the kernel of $S$, $E(M)=\{g\}$. By Corollary 1.4, $g \in C(E(S))=\Lambda$. Let $\Omega=\Omega(S)$. Let $S_{\alpha}(\alpha \in \Omega)$ be the maximal semilattice decomposition of $S$ and let $\rho$ be the corresponding congruence. By Tamura [14], each $S_{\alpha}$ is $\delta$-indecomposable. By Lemma 1.6, each $S_{\alpha}$ is strongly $\pi$-regular, has a kernel $M_{\alpha}$ and $M_{\alpha} \in \mathcal{U}(S)$. Let $e_{\alpha} \in E\left(M_{\alpha}\right)$. Since $M_{\alpha}^{2} \subseteq M_{\alpha}$, we see by Theorem 2.10 that $e_{\alpha} \in \Lambda$ and that $M_{\alpha}$ is a group. Suppose $f \in \Lambda \cap S_{\alpha}$. Then $f \in C\left(S_{\alpha}\right)$. So $I=f S_{\alpha}=S_{\alpha} f$ is an ideal of $S_{\alpha}$ and hence by Tamura [14], is $\mathcal{S}$-indecomposable. Since $S_{\alpha}$ is strongly $\pi$-regular, so is $I$. Since $I$ has an identity element $f$, we see by Lemma 1.5 , that $I$ is a group. Hence $M_{\alpha}=I$ and $f=e_{\alpha}$. Then $\Lambda \cap S_{\alpha}=\left\{e_{\alpha}\right\}$ for all $\alpha \in \Omega$. Hence $\Lambda=\left\{e_{\alpha} \mid \alpha \in \Omega\right\}$ and $\Lambda \cong$ $\Omega$. Let $a, b \in S$ such that $a \delta b$. Let $a \in S_{\alpha}, b \in S_{\beta}$. Then $a e_{\alpha} \in M_{\alpha}$ and $a e_{\alpha} \mathcal{H} e_{\alpha}$. Hence $e_{\alpha} \in N(a)=N(b)$. So be $e_{\alpha} \mathcal{H} e_{\alpha}$. Thus $b e_{\alpha} \in S_{\alpha}$ and $\beta \geqslant \alpha$. Similarly, $\alpha \geqslant \beta$ and $\alpha=\beta$. Hence $a \rho b$. Conversely, let $a, b \in S$ such that $a \rho b$. Then $a, b \in S_{\alpha}$ for some $\alpha \in \Omega$. Suppose $f \in N(a)$. Then $f=e_{\beta}$ for some $\beta \in \Omega$ and $a e_{\beta} \mathcal{F} e_{\beta}$. Hence 
$a e_{\beta} \in S_{\beta}$ and $\alpha \geqslant \beta$. So $b e_{\beta} \in S_{\beta}$. Thus $\left(b e_{\beta}\right) e_{\beta} \in M_{\beta}$ and $b e_{\beta} \mathcal{H} e_{\beta}$. Hence $f=e_{\beta}$ $\in N(b)$. So $N(a) \subseteq N(b)$. Similarly, $N(b) \subseteq N(a)$ and $N(a)=N(b)$. Hence $a \delta b$. Thus $\rho=\delta$, proving the theorem.

THEOREM 2.12. Let $S$ be a connected regular semigroup. Then the following conditions are equivalent.

(1) $\mathcal{U}(S)$ is linearly ordered.

(2) All ideals of $S$ are connected.

Proof. (1) $\Rightarrow(2)$. If $\mathscr{U}(S)$ is linearly ordered, then every ideal of $S$ is of the form SeS, $e \in E(S)$ and we are done by Corollary 2.5 .

$(2) \Rightarrow(1)$. Let $e, f \in E(S)$ and let $I=S e S \cup S f S$. $I$ is an ideal of $S$ and hence connected. By Theorem 2.7, there exists $g \in E(T)$ such that $I g I=I$. So $g|e, g| f$. Now $g \in \operatorname{SeS}$ or $g \in S f S$. By symmetry, assume $g \in \operatorname{SeS}$. Then $e|g| f$ and $e \mid f$. Hence $\mathscr{U}(S)$ is linearly ordered.

THEOREM 2.13. Let $S$ be a connected regular semigroup such that $\mathscr{U}(S)$ is linearly ordered. Then:

(1) $|\Omega(S)| \leqslant 2$.

(2) Suppose $|\Omega(S)|=2$. Then $S$ has a unique proper prime ideal $P$ such that $S \backslash P$ is completely simple. If further the kernel of $S$ is a group, then $S \backslash P$ is a group and $S$ has an identity element.

Proof. Suppose $|\Omega(S)| \neq 1$ and let $P$ be a proper prime ideal of $S$. Then $S \backslash P$ is strongly $\pi$-regular. Let $e \in E(S \backslash P)$. We claim that $S e S=S$. Suppose $S e S \neq S$. Since $\mathcal{U}(S)$ is linearly ordered, there exists $f \in E(S)$ such that $P=S f S$ and $e \mid f$. Let $p=\operatorname{dim} \cdot S f S, m=\operatorname{dim} \cdot \operatorname{Ses}, n=\operatorname{dim} \cdot S$. Then $p<m<n$. Define $\phi: S \times S$ $\rightarrow \operatorname{SeS}$ as $\phi(x, y)=x e y$. Then $\phi$ is surjective. Since $e \mid f, \phi(P \times P)=P$. Let $W$ be an irreducible component of $\phi^{-1}(P)$ containing the irreducible closed set $P \times P$. Then $\phi(W)=P$. Hence by $[9$, Theorem 4.1], $\operatorname{dim} \cdot W \geqslant \operatorname{dim} \cdot P+\operatorname{dim} \cdot S \times S$ $-\operatorname{dim} S e S=p+2 n-m>n+p$. Now $\operatorname{dim} \cdot((P \times S) \cup(S \times P))=n+p$. Hence $W \nsubseteq(P \times S) \cup(S \times P)$. Thus there exist $a, b \in S \backslash P$ such that $(a, b)$ $\in W$. Hence aeb $\in P, a, e, b \in S \backslash P$. This contradicts the fact that $P$ is a prime ideal of $S$. Hence $S e S=S, S \backslash P$ is completely simple, and $S \backslash P$ is the maximum element of $U(S)$. If $P_{1}$ is any other proper prime ideal of $S$ then $S \backslash P=S \backslash P_{1}$ and hence $P=P_{1}$. Thus $P$ is the unique prime ideal of $S$. Since $\Omega(S)$ is a homomorphic image of $S, \Omega(S)$ cannot have more than one proper prime ideal. Let $\alpha_{1}, \alpha_{2} \in \Omega=\Omega(S)$ such that $\alpha_{i}$ is not the minimum element of $\Omega, i=1,2$. Let $\Omega_{i}=\left\{\beta \mid \beta \in \Omega, \beta \neq \alpha_{i}\right\}, i=1,2$. Then $\Omega_{i}$ is a proper prime ideal of $\Omega, i=1,2$. So $\Omega_{1}=\Omega_{2}$ and $\alpha_{1}=\alpha_{2}$. Then $|\Omega|=2$. Next assume that the kernel of $S$ is a group. Then by Theorem 2.10,e $e(S)$, and $S \backslash P$ is a group. So $S=S e S=e S=S e$ and $e$ is the identity element of $S$. This proves the theorem.

3. Clifford semigroups. A semigroup $S$ is said to be Clifford semigroup if it is a union of its subgroups. Clifford semigroups were first studied by Clifford [2]. For the basic results about Clifford semigroups, see Clifford.[2] or [3, Theorem 4.6]. In 
particular, each $g$-class of $S$ is a subsemigroup and for all $J_{1}, J_{2} \in \mathcal{Q}(S), J_{1} J_{2} \subseteq$ $J_{1} \wedge J_{2}$. If $S$ is a Clifford semigroup and $a \in S$, then we let $a^{-1}$ denote the inverse of $a$ in the maximal subgroup of $a$. If each $g$-class of $S$ is a subgroup then $S$ is a semilattice of groups. Semilattices of groups were first studied by Clifford [2]. In particular, every idempotent of a semilattice of groups $S$ is contained in the center of $S$. A regular semigroup $S$ in which idempotents commute is said to be an inverse semigroup. By the author [11, Theorem 2.8], a connected semigroup is inverse if and only if it is a semilattice of groups. Hence by Theorem 2.7, a connected inverse semigroup has an identity element.

A lattice $L=(L, \wedge, \bigvee)$ with a maximum element 1 and a minimum element 0 is complemented if for all $e \in L$, there exists $f \in L$ such that $e \vee f=1$ and $e \wedge f=$ 0 . $L$ is relatively complemented if for all $e, f \in L$ with $e<f,[e, f]=\{x \mid x \in L$, $e \leqslant x \leqslant f\}$ is complemented. It is well known [4] that a complemented modular lattice is relatively complemented. For finite lattices, the situation is clearly the other way around, i.e. every finite relatively complemented lattice is complemented.

LEMMA 3.1. Let $S$ be an algebraic Clifford semigroup, $T$ a closed subsemigroup of $S$. Then $T$ is a Clifford semigroup.

Proof. Since $T$ is an algebraic semigroup, $T$ is strongly $\pi$-regular. Let $a \in T$. Then $a^{n} \mathcal{H} e$ in $T$ for some $e \in E(T), n \in \mathbf{Z}^{+}$. Since $S$ is a Clifford semigroup, $a \mathcal{H} f$ in $S$ for some $f \in E(S)$. Then $e \mathcal{H} a^{n} \mathcal{H} f$ in $S$ and so $e=f$. Thus $e a=a e=a$. Since $a^{n} \mathcal{H} e$ in $T$, we now see that $a \mathcal{H} e$ in $T$. Thus $T$ is a Clifford semigroup.

By Theorem 2.10 we have

TheOREM 3.2. Let $S$ be a connected Clifford semigroup. Then the following conditions are equivalent.

(1) The kernel of $S$ is a group.

(2) $S$ is a semilattice of groups.

LemMa 3.3. Let $S$ be a connected Clifford semigroup, $f \in E(S)$ such that $S f S \neq S$. Define $\phi: S \rightarrow S \times S$ as $\phi(x)=(f x, x f)$. Let $V=\overline{\phi(S)}$. Then $\operatorname{dim} \cdot V<\operatorname{dim} \cdot S$.

Proof. Let $T=S f S$. Then $T$ is a connected Clifford semigroup and $\operatorname{dim} \cdot T<$ $\operatorname{dim} \cdot S$. We claim that $\phi(S)=\phi(T)$. Let $x \in S$. Then $f x \Re f x f \mathcal{L} x f$. Let $f x f \mathcal{H} e$, $e \in E(T)$. Then $f \geqslant e$ and so $f a=a f=a$ for all $a \in H_{e}$. Since $f x \mathcal{R} e$, $e f x=f x$. Similarly, $x f e=x f$. Let $y=x(f x f)^{-1} x \in T$. Now $f(f x f)^{-1}=(f x f)^{-1} f=(f x f)^{-1}$. So $f y=f x f(f x f)^{-1} f x=e f x=f x$. Similarly, $y f=x f$ and $\phi(y)=\phi(x)$. Since $y \in T$ we see that $\phi(S)=\phi(T)$. Hence $V=\overline{\phi(T)}$ and $\operatorname{dim} \cdot V \leqslant \operatorname{dim} \cdot T<\operatorname{dim} \cdot S$.

If $S, T$ are algebraic semigroups, then $S \mid T\left(S\right.$ divides $T$ ) if $S$ is a ${ }^{*}$-homomorphic image of a closed subsemigroup of $T$.

THEOREM 3.4. Let $S$ be a connected Clifford semigroup, $J_{1}, J_{2} \in \mathcal{Q}(S), J_{1}>J_{2}$. Let $G_{i}$ be the maximal subgroup of $J_{i}$ with identity element $e_{i}, i=1,2$. Then:

(1) For each $g \in E\left(J_{2}\right)$, there exists $f \in E\left(J_{1}\right)$ such that $f \geqslant g$.

(2) $\tilde{G}_{2} \mid \tilde{G}_{1}$.

(3) Suppose $S$ is an inverse semigroup and define $\psi: \tilde{G}_{1} \rightarrow \tilde{G}_{2}$ as $\psi\left(a, a^{-1}\right)=$ $\left(e_{2} a, e_{2} a^{-1}\right)$. Then $\psi$ is a surjective *-homomorphism. 
Proof. We can assume without loss of generality (since $U(S)$ is finite) that $J_{1}$ covers $J_{2}$. Let $e \in E\left(J_{1}\right), g \in E\left(J_{2}\right)$. We can assume that $S e S=S$ (otherwise we work with $S e S)$. Clearly $S g S \neq S$. Define $\phi: S \rightarrow S \times S$ as $\phi(x)=(g x, x g)$ and let $V=\overline{\phi(S)}$. By Lemma 2.3, $\operatorname{dim} \cdot V<\operatorname{dim} \cdot S$. Consider $\phi: S \rightarrow V$. Then $V$ is irreducible and $\phi$ is a dominant morphism. Clearly $\phi(g)=(g, g)$. Hence [9, Theorem 4.1], $\phi^{-1}(g, g) \neq\{g\}$. So there exists $x \in S$ such that $x \neq g$ and $x g=g x$ $=g$. Suppose $x \in J_{2}$. Then $x \mathcal{H} g$ and so $x=g$, a contradiction. So $x \notin J_{2}$. Since $e|x| g$ and $J_{1}$ covers $J_{2}, x \in J_{1}$. Let $x \mathcal{H} f, f \in E\left(J_{1}\right)$. Then $f \geqslant g$. This proves (1). We now prove (2) and (3). By Lemma 1.2 we can assume, without loss of generality, that $e_{1}=f$ and $e_{2}=g$. Let $G=\left\{a \mid a \in G_{1}, a g=g a\right\}, \tilde{G}=\left\{\left(a, a^{-1}\right) \mid a\right.$ $\in G\}$. Then $G$ is a subgroup of $G_{1}$ and $\tilde{G}$ is a subgroup of $\tilde{G}_{1}$. Note that if $S$ is an inverse semigroup, then $G=G_{1}$ and $\tilde{G}=\tilde{G}_{1}$. Define $\lambda: G \rightarrow G_{2}$ as $\lambda(a)=a g=g a$ and define $\psi: \tilde{G} \rightarrow \tilde{G}_{2}$ as $\psi\left(a, a^{-1}\right)=\left(\lambda(a), \lambda\left(a^{-1}\right)\right)$. Then $\lambda$ is a homomorphism and $\psi$ is a ${ }^{*}$-homomorphism. It suffices to show that $\psi$ is surjective. This is equivalent to showing that $\lambda$ is surjective. Let $b \in G_{2}$. Then $\phi(b)=(b, b)$. So as above, $\phi^{-1}(b, b)$ $\neq\{b\}$. Hence there exists $y \in S, y \neq b$ such that $y g=g y=b$. Since $f \geqslant g$, $b f=f b=b$. If $y \in J_{2}$ then $y \in G_{2}$ and so $y=b$, a contradiction. Hence $y \notin J_{2}$. Since $e|y| b$ and $J_{1}$ covers $J_{2}, y \in J_{1}$. Then $a=f y f \in G_{1}$. Now $g a=g f y f=g y f=$ $b f=b$. Similarly $a g=b$. Hence $a \in G$ and $\lambda(a)=b$. Thus $\lambda$ is surjective, proving the theorem.

Corollary 3.5. Let $S$ be a connected Clifford semigroup, e, $f, g \in E(S), e|f| g$, $e \geqslant g$. Then there exists $h \in E(S)$ such that $h g f$ and $e \geqslant h \geqslant g$.

Proof. By Lemma 1.3, there exists $f_{1} \in E(S)$ such that $e \geqslant f_{1}, f_{1} g f$. So $e, f_{1}, g \in$ $T=e S e$. By Lemma $1.7, f_{1} \mid g$ in $T$. So by Theorem 3.4, there exists $h \in E(T)$ such that $h g_{f_{1}}$ and $h \geqslant g$. Since $h \in T, e \geqslant h$. Also $h g_{f_{1}} g f$.

LEMMA 3.6. Let $S$ be a connected Clifford semigroup, e,f $\in E(S)$ such that $e$ covers $f$. Then there is a closed connected subsemigroup $T$ of $S$ such that $e, f \in T$ and af $=$ fa $=$ f for all $a \in T$.

Proof. We can assume without loss of generality that $e=1$ is the identity element of $S$ (otherwise we work with $e S e$ ). Clearly $S f S \neq S$. Define $\phi: S \rightarrow S \times S$ as $\phi(x)=(f x, x f)$. Let $V=\overline{\phi(S)}$. By Lemma 3.3, dim $\cdot V<\operatorname{dim} \cdot S$. The map $\phi$ : $S \rightarrow V$ is dominant and $(f, f)=\phi(f) \in \phi(S)$. Let $A=\phi^{-1}(f, f)$. Then $A$ is a closed subsemigroup of $S, 1, f \in A$. Let $B$ be an irreducible component of $A$ such that $f \in B$. By [9, Theorem 4.1], $\operatorname{dim} \cdot B>0$. Hence there exists $x \in B, x \neq f$. So $x f=f x=f$. Hence $x \mathscr{X} f$. Let $x \mathcal{H} g, g \in E(\dot{A})$. Then $1 \geqslant g>f$. Since 1 covers $f$, $1=g$. Let $X$ be the irreducible component of $A$ containing $x^{-1}$. Define $\psi$ : $X \times B \rightarrow A$ as $\psi(c, d)=c d$. Since $X \times B$ is irreducible, $\psi(X \times B)$ is contained in an irreducible component $T$ of $A$. Now $1=x^{-1} x=\psi\left(x^{-1}, x\right) \in \psi(X \times B) \subseteq T$ and $f=x^{-1} f=\psi\left(x^{-1}, f\right) \in \psi(X \times B) \subseteq T$. Hence $1, f \in T$. By Lemma $1.9, T$ is a subsemigroup of $S$.

THEOREM 3.7. Let $S$ be a connected Clifford semigroup. Then for any $f \in E(S)$, the set $\{e \mid e \in E(S), e \geqslant f\}$ is finite. 
Proof. We prove the theorem by induction on $\operatorname{dim} \cdot S$. For $e, f \in E(S)$, set $M_{e, f}=\left\{g \mid g \in E(S), g \geqslant f, g g_{e}\right\}$. Since $\mathcal{Q}(S)$ is finite, it suffices to show that $M_{e, f}$ is finite for all $e, f \in E(S)$. So suppose $M_{e, f}$ is infinite for some $e, f \in E(S)$. Of all such possibilities, choose one with $J_{e}$ minimal. For that $e$ choose $f$ with $J_{f}$ maximal. Since $M_{e, f}$ is infinite, we see that $J_{e}>J_{f}$. We claim that $J_{e}$ covers $J_{f}$. For suppose there exists $d \in E(S)$ such that $J_{e}>J_{d}>J_{f}$. By the minimality of $J_{e}, M=M_{d, f}$ is finite. By the maximality of $J_{f}, M_{e, h}$ is finite for all $h \in E\left(J_{d}\right)$. Let $g \in M_{e, f}$. Then $g|d| f$ and $g \geqslant f$. By Corollary 3.5, there exists $h \in M$ such that $g \in M_{e, h}$. Hence $M_{e, f} \subseteq \cup_{h \in M} M_{e, h}$. Thus $M_{e, f}$ is finite, a contradiction. It follows that $J_{e}$ covers $J_{f}$. We can also assume that $e \geqslant f$. Otherwise we can replace $e$ by any $e_{1} \in M_{e_{f}}$.

Now assume that $e S \neq S$ and $S e \neq S$. Then $e S$ and $S e$ are connected Clifford semigroups and $\operatorname{dim} \cdot e S<\operatorname{dim} \cdot S, \operatorname{dim} \cdot S e<\operatorname{dim} \cdot S$. By our induction hypothesis, the sets $A_{1}=M_{e, f} \cap e S$ and $A_{2}=M_{e, f} \cap S e$ are finite. If $g \in A_{1}$ and

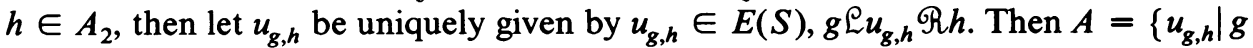
$\left.\in A_{1}, h \in A_{2}\right\}$ is finite. Let $g_{1} \in M_{e, f}$. Since $g_{1} g e$, there exist $g, h \in E(S)$ such

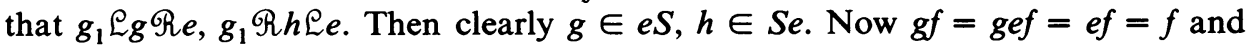
$f g=f g_{1} g=f g_{1}=f$. Hence $g \in M_{e_{f}}$. Similarly $h \in M_{e_{f}}$. Hence $g \in A_{1}, h \in A_{2}$.

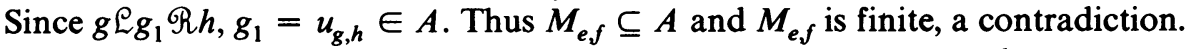

We can therefore assume by symmetry that $e S=S$. Hence the $g$-class of $e$ is an $\Re$-class and $g S=S$ for all $g \in M_{e, f}$. Let $T=\{a \mid a \in S$, af $=f a=f\}$. Then $T$ is a closed Clifford subsemigroup of $S, M_{e, f} \subseteq T$ and $f \in T$. Let $T_{1}, \ldots, T_{k}$ be the irreducible components of $T$. Let $g \in M_{e, f}$. We claim that $g$ covers $f$ in $E(S)$. For suppose $g>f^{\prime}>f$ for some $f^{\prime} \in E(S)$. Then $J_{e}>J_{f^{\prime}}>J_{f}$, contradicting the fact that $J_{e}$ covers $J_{f}$. Then $g$ covers $f$. By Lemma 3.6, there exists a closed connected subsemigroup $B(g)$ of $S$ such that $f, g \in B(g)$ and $a f=f a=f$ for all $a \in B(g)$. Hence $B(g) \subseteq T$. Therefore $B(g) \subseteq T_{r}$ for some $r$. Let $\psi(g)=T_{r}$. Thus $\psi: M_{e, f} \rightarrow$ $\left\{T_{1}, \ldots, T_{k}\right\}$ and $f, g \in \psi(g)$ for all $g \in M_{e_{f}}$. Since $M_{e_{f}}$ is infinite, there exist $g_{1}, g_{2} \in M_{e, f}$ such that $g_{1} \neq g_{2}$ and $\psi\left(g_{1}\right)=\psi\left(g_{2}\right)=T_{i}$. So $f, g_{1}, g_{2} \in T_{i} . T_{i}=g_{1} T_{i}$ $\subseteq T_{i} T_{i}$. Since $T_{i} T_{i}$ is the morphic image of the irreducible set $T_{i} \times T_{i}$, it follows that $T_{i} T_{i}$ is irreducible. Hence $T_{i}=T_{i} T_{i}$. Thus $T_{i}$ is a connected Clifford semigroup. Since $T_{i} \subseteq T, f$ is the zero of $T_{i}$. By Theorem 3.2, $T_{i}$ is a semilattice of groups. In particular, $g_{1} g_{2}=g_{2} g_{1}$. Hence $g_{1}=g_{2}$, a contradiction. This contradiction proves the theorem.

LEMMA 3.8. Let $S$ be a connected Clifford semigroup. Let $J_{1}, J_{2} \in \mathcal{U}(S)$ such that $J_{1}>J_{2}$ and $J_{1}$ is not the maximum element of $\mathcal{Q}(S)$. Then there exists $J_{3} \in \mathcal{Q}(S)$ such that $J_{3}>J_{2}$ and $J_{1} \wedge J_{3}=J_{2}$.

Proof. Let $f \in E\left(J_{1}\right)$. By Lemma 1.3, there exists $g \in E\left(J_{2}\right)$ such that $f \geqslant g$. Clearly $S f S \neq S$. Define $\phi: S \rightarrow S \times S$ as $\phi(x)=(f x, x f)$ and let $V=\overline{\phi(S)}$. By Lemma $3.3, \operatorname{dim} \cdot V<\operatorname{dim} \cdot S$. $\phi$, considered as a morphism from $S$ into $V$, is dominant. Since $\phi(g)=(g, g)$ we see by $\left[9\right.$, Theorem 4.1] that $\phi^{-1}(g, g) \neq\{g\}$. So there exists $x \in S, x \neq g$ such that $f x=x f=g$. Hence $g x=x g=g$. If $x g g$, then $x \mathcal{H} g$ and so $x=g$, a contradiction. Hence $J_{x} \neq J_{2}$ and $J_{1} \wedge J_{x}=J_{2}$. 
LEMMA 3.9. Let $S$ be a connected Clifford semigroup with an identity element 1 . Let $h \in E(S)$. Then there exists a connected closed subsemigroup $T$ of $S$ such that 1 , $h \in T, h$ is the zero of $T$ and for all $J \in \mathcal{U}(S), J \geqslant J_{h}$ implies that $J \cap T$ is $a$ subgroup of $T$.

Proof. Let $G$ be the group of units of $S$ and set $B=\{a \mid a \in S, a h=h a=h\}$. Clearly $1, h \in B$ and $B$ is a closed subsemigroup of $S$. By Lemma 1.9, 1 lies in a unique irreducible component $T$ of $B$ such that $T$ is a subsemigroup of $B$. Let $\mathbb{Q}=\left\{J \mid J \in \mathcal{Q}(S), J \geqslant J_{h}\right\}$. We claim that $T \cap J \neq 0$ for all $J \in \mathbb{Q}$. Clearly $G \cap T \neq \varnothing$. Suppose $T \cap J=\varnothing$ for some $J \in \mathbb{Q}$. Choose $J$ maximal. Suppose $G$ does not cover $J$ in $\mathcal{Q}(S)$. Then there exists $J_{1} \in \mathcal{U}(S)$ such that $G>J_{1}>J$. By Lemma 3.8, there exists $J_{2} \in \mathcal{Q}(S)$ such that $J_{2}>J$ and $J_{1} \wedge J_{2}=J$. Clearly $J_{1}, J_{2} \in Q$. By the maximality of $J, J_{1} \cap T \neq \varnothing$ and $J_{2} \cap T \neq 0$. Since $J_{1} J_{2} \subseteq J_{1}$ $\wedge J_{2}=J$, we see that $J \cap T \neq \varnothing$, a contradiction. Hence $G$ covers $J$. By Theorem 3.4 , there exists $g \in E(J)$ such that $g \geqslant h$. Clearly 1 covers $g$ in $E(S)$. By Lemma 3.6, there exists a closed connected subsemigroup $A$ of $S$ such that $1, g \in A$ and $a g=g a=g$ for all $a \in A$. Since $g \geqslant h, a h=h a=h$ for all $a \in A$. Hence $A \subseteq B$. Since $1 \in A, A \subseteq T$. Hence $g \in J \cap T$, a contradiction. Thus $T \cap J \neq \varnothing$ for all $J \in \mathbb{Q}$. In particular $J_{h} \cap T \neq \varnothing$. Let $a \in J_{h} \cap T$. By Lemma 3.1, $a \mathcal{F} h_{1}$ for some $h_{1} \in E(T)$. So $h_{1} g h$. Since $T \subseteq B, h_{1} \geqslant h$ and $h_{1}=h$. Thus $h$ is the zero of $T$. By Theorem 3.2, $T$ is semilattice of groups. Let $a, b \in T$ such that $a g b$ in $S$. There exist $e, f \in E(T)$ such that $a \mathcal{H} e$ and $b \mathcal{H} f$ in $T$. Hence $f g=g f$ and $f g g$ in $T$. So $f=g$ and $a \mathcal{H} b$ in $T$. Hence $\mathcal{Q}(T)=\{J \cap T \mid J \in \mathbb{Q}\}$, proving the lemma.

As usual $\operatorname{GL}(n, K)$ will denote the algebraic group $\left\{(a, 1 / \operatorname{det} \cdot a) \mid a \in \mathfrak{T}_{n}(K)\right.$, $\operatorname{det} \cdot a \neq 0\}$. If $a \in \operatorname{GL}(n, K)$, then $a$ is semisimple if it is diagonalizable. $a$ is unipotent if its sole eigenvalue is 1 . These definitions generalize to any algebraic group (see [9, Chapter VI]). If $G$ is an algebraic group, then let $G_{s}=\{a \mid a \in G, a$ is semisimple $\}, G_{u}=\{a \mid a \in G, a$ is unipotent $\}$. $G$ is unipotent if $G_{u}=G$. Suppose $G_{s}=G$ and let $\phi: G \rightarrow \Re_{n}(K)$ be a *-homomorphism. Let $e$ be the identity element of $G$. We can assume without loss of generality that

$$
\phi(e)=\left(\begin{array}{ll}
I_{r} & 0 \\
0 & 0
\end{array}\right)
$$

where $I_{r}$ is the $r \times r$ identity matrix. If $a \in G$, then

$$
\phi(a)=\left(\begin{array}{ll}
A & 0 \\
0 & 0
\end{array}\right)
$$

where $A$ is an $r \times r$ invertible matrix. Set $\psi(a)=(A, 1 / \operatorname{det} \cdot A)$. Thus $\psi: G \rightarrow$ $\mathrm{GL}(r, K)$ is a *-homomorphism. Hence by [9, Theorem 15.3], every element of $\psi(G)$ is semisimple. Hence every element of $\phi(G)$ is diagonalizable. Thus we have

LeMma 3.10. Let $G$ be an algebraic group, $G_{s}=G, \phi: G \rightarrow \mathscr{T}_{n}(K) a^{*}$-homomorphism. Then every element of $\phi(G)$ is diagonalizable.

We let $K^{+}$denote the algebraic group $(K,+)$ and $K^{*} \subseteq\left(K^{2}, \cdot\right)$ the algebraic group $\{(a, b) \mid a, b \in K, a b=1\}$. 
LEMMA 3.11. Let $S$ be a connected semigroup with identity element 1. Suppose $S=G \cup\{0\}$ where $G$ is the group of units of $S$ and 0 is the zero of $S$. Then $\operatorname{dim} \cdot S=1, \tilde{G}_{s}=\tilde{G}, \tilde{G}$ is ${ }^{*}$-isomorphic to $K^{*}$ and $S$ is commutative.

Proof. By [5, II, §2, Theorem 3.3] we can assume that $S$ is a closed submonoid of $\Re_{n}(K)$ for some $n \in Z^{+}$. Then $G=\{a \mid a \in S$, det $\cdot a \neq 0\}$. Define $\phi: S \rightarrow K$ as $\phi(a)=\operatorname{det} \cdot a$. The map $\phi: S \rightarrow \overline{\phi(S)}$ is dominant and $\phi^{-1}(0)=\{0\}$ we see by $[9$, Theorem 4.1] that $\operatorname{dim} \cdot S=1$. Since $S$ is connected, $\phi(S) \neq\{1,0\}$. So $\phi(G) \neq$ $\{1\}$. By Lemma 1.1, dim $\cdot \tilde{G}=1$. Consider the *-homomorphism $\psi: \tilde{G} \rightarrow K^{*}$ given by $\psi\left(a, a^{-1}\right)=\left(\phi(a), \phi\left(a^{-1}\right)\right)$. Then $|\psi(\tilde{G})|>1$. Now by [9, Theorem 20.5], $\tilde{G}$ is either ${ }^{*}$-isomorphic to $K^{*}$ or $K^{+}$. The second possibility is ruled out by [9, Theorem 15.3] since $|\psi(\tilde{G})|>1$. Hence $\tilde{G}$ is *-isomorphic to $K^{*}$ and $\tilde{G}_{s}=\tilde{G}$. In particular, $G$ (and hence $S$ ) is commutative.

THEOREM 3.12. Let $S$ be a connected Clifford semigroup with kernel $M$. Then the following conditions are equivalent.

(1) Some $G$-class $J$ of $S$ is commutative.

(2) $M$ is commutative.

(3) $S$ is commutative.

Proof. First assume (1). Let $J \in \mathcal{U}(S)$ be commutative and let $e \in E(J)$. Then $J$ is a group and by Lemma 1.10,e $e C(S)$. So $M \subseteq T=e S=S e$. Let $G$ be the group of units of $T$. Then $G=J$ is abelian. So by Lemma 1.5 and [11, Theorem 2.4], $e T e=T$ is commutative. Hence $M$ is commutative and (2) holds.

Next assume (2). Then $M$ is a group. By Theorem 3.2, $S$ is a semilattice of groups. Let 1 be the identity element of $S$ and let $G$ be the group of units of $S$. We prove $(2) \Rightarrow(3)$ by induction on $\operatorname{dim} \cdot S$. Let 1 cover $e \in E(S)$. Then by our induction hypothesis, $e S$ is commutative. Let $T=\{a \mid a \in S, a e=e\}$. By Lemma 1.9, 1 lies in a unique irreducible component $T_{1}$ of $T$ and $T_{1}^{2} \subseteq T_{1}$. By Lemma 3.6, $e \in T_{1}$. So $E\left(T_{1}\right)=\{1, e\}$ and $e$ is the zero of $T_{1}$. So $H=T_{1} \backslash\{e\}$ is a subgroup of $G$. By Lemma 3.11, $T_{1}$ is commutative and $\tilde{H}_{s}=\tilde{H}$. Let $\theta: \tilde{G} \rightarrow S$ be the *-homomorphism $\theta\left(a, a^{-1}\right)=a$. Let $G_{1}$ be the group of units of $e S$. Then $G_{1}$ is abelian. Define $\phi: \tilde{G} \rightarrow \tilde{G}_{1}$ by $\phi\left(a, a^{-1}\right)=\left(e a, e a^{-1}\right)$. Let $N$ denote the kernel of $\phi$. Then $\theta(N) \subseteq T$. Since $\tilde{G}_{1}$ is abelian, $(\tilde{G}, \tilde{G}) \subseteq N$ where $(\tilde{G}, \tilde{G})$ is the commutator subgroup of $\tilde{G}$. By [9, Proposition 17.2], $(\tilde{G}, \tilde{G})$ is closed and connected. Since $1 \in \theta(\tilde{G}, \tilde{G}), \theta(\tilde{G}, \tilde{G}) \subseteq T_{1}$ and so $\theta(\tilde{G}, \tilde{G}) \subseteq H$. Thus $(\tilde{G}, \tilde{G}) \subseteq \tilde{H}$ and $(\tilde{G}, \tilde{G})$ is abelian. In particular, $\tilde{G}$ is solvable. By [9, Theorem 19.3], $(\tilde{G}, \tilde{G}) \subseteq \tilde{G}_{u}$. Since $\tilde{H}_{s}=\tilde{H}$, we see that $|(\tilde{G}, \tilde{G})|=1$. Hence $\tilde{G}$ is abelian. Thus $G$ is abelian. By [11, Theorem 2.4], $S$ is commutative. Thus (3) holds. That $(3) \Rightarrow(1)$ is trivial.

LEMMA 3.13. Let $S$ be a commutative connected Clifford semigroup, e, $f \in E(S)$ such that $e>f$. Let $G, H$ denote the $\mathcal{H}$-classes of $e$ and $f$ respectively. If $\tilde{H}_{s}=\tilde{H}$, then $\tilde{G}_{s}=\tilde{G}$.

Proof. Since $E(S)$ is finite, we are easily reduced to the case when $e$ covers $f$. We can assume that $e=1$ is the identity element of $S$ (otherwise we work with $e S$ ). Let $T=\{a \mid a \in S$, af $=f\}, T_{1}$ the (unique) irreducible component of $T$ containing 
1. Then as in the proof of Theorem 3.12, $f \in T_{1}$ and $\tilde{W}_{s}=\tilde{W}$ where $W=T_{1} \backslash\{f\}$ $\subseteq G$. Consider the *-homomorphism $\phi: \tilde{G} \rightarrow \tilde{H}$ given by $\phi(a)=\left(a f, a^{-1} f\right)$. Let $N$ denote the kernel of $S$. If $\theta: \tilde{G} \rightarrow S$ is given by $\theta\left(a, a^{-1}\right)=a$, then $\theta(N) \subseteq T$. Since $\tilde{H}_{s}=\tilde{H}$ we see by $\left[9\right.$, Theorem 15.3] that $\tilde{G}_{u} \subseteq N$. By [9, Theorem 15.5] and Lemma $1.1, \tilde{G}_{u}$ is closed and connected. Since $1 \in \theta\left(\tilde{G}_{u}\right)$ we see that $\theta\left(\tilde{G}_{u}\right) \subseteq T_{1}$. Thus $\theta\left(\tilde{G}_{u}\right) \subseteq W$ and $\tilde{G}_{u} \subseteq \tilde{W}$. Since $\tilde{W}_{s}=\tilde{W}$ we see that $\left|\tilde{G}_{u}\right|=1$. Hence $\tilde{G}_{s}=\tilde{G}$.

An algebraic semigroup $S$ is a $d$-semigroup if $S$ is *-isomorphic to a closed subsemigroup of $\left(K^{n}, \cdot\right)$ for some $n \in Z^{+}$. By Lemma 3.1, every $d$-semigroup is a commutative Clifford semigroup.

THEOREM 3.14. Let $S$ be a connected Clifford semigroup with kernel $M$. Then $S$ is a $d$-semigroup if and only if $M$ is a d-group.

Proof. If $S$ is a $d$-semigroup then clearly $M$ is a $d$-group. So assume conversely that $M$ is a $d$-group. Then by Theorem $3.12, S$ is commutative. $\tilde{M}$ is a $d$-group and hence $\tilde{M}_{s}=\tilde{M}$. We see by Lemma 3.13 that $\tilde{G}_{s}=\tilde{G}$ for all maximal subgroups $G$ of $S$. By [11, Corollary 1.3], we can assume that $S$ is a closed subsemigroup of $\mathscr{T}_{n}(K)$ for some $n \in \mathbf{Z}^{+}$. Let $a \in S$. Then $a \in G$ for some maximal subgroup $G$ of $S$. Consider $\phi: \tilde{G} \rightarrow S$ given by $\phi\left(b, b^{-1}\right)=b$. Since $a \in \phi(\tilde{G})$ and $\tilde{G}_{s}=\tilde{G}$, we see by Lemma 3.10 that $a$ is diagonalizable. Thus every element of $S$ is diagonalizable. Since $S$ is commutative it follows $[8, \S 6.5$, Theorem 8$]$ that there exists an invertible matrix $c \in \mathscr{N}_{n}(K)$ such that $c S c^{-1}$ consists of diagonal matrices. This proves the theorem.

COROLlary 3.15. Let $S$ be a connected Clifford semigroup with a zero. Then $S$ is a d-semigroup.

Theorem 3.16. Let $S$ be a connected Clifford semigroup. Then there exists a closed connected subsemigroup $T$ of $S$ such that $T$ is a d-semigroup with zero and $T \cap J$ is $a$ subgroup of $T$ for all $J \in \mathcal{U}(S)$. In particular $T$ is a semilattice union of the groups $T \cap J, J \in \mathcal{Q}(S)$.

Proof. By Theorem 2.7, there exists $e \in E(S)$ such that $S e S=S$. Let $J \in$ $\mathcal{Q}(S)$. By Lemma 1.3, there exists $f \in E(J)$ such that $f \in e S e$. Hence by Lemma 1.7, $\mathcal{U}(e S e)=\{J \cap e S e \mid J \in \mathcal{U}(S)\}$. Thus, without loss of generality, we can assume that $e=1$ is the identity element of $S$. Let $M$ be kernel of $S$ and let $h \in E(M)$. By Lemma 3.9, there exists a closed connected subsemigroup $T$ of $S$ with zero $h$ such that $T \cap J$ is a subgroup of $T$ for all $J \in \mathcal{Q}(S)$. By Corollary $3.15, T$ is a $d$-semigroup.

THEOREM 3.17. Let $S$ be a connected inverse semigroup with kernel $M$. Let $\Lambda$ be any maximal chain in $E(S)$. Then $\operatorname{dim} \cdot S=\operatorname{dim} \cdot M+|\Lambda|-1$.

Proof. We prove by induction on $\operatorname{dim} \cdot S=n$. Let $\Lambda=\left\{e_{1}<e_{2}<\cdots<e_{k}\right\}$ be a maximal chain in $E(S)$. If $k=1$, then $S$ is a group and there is nothing to prove. So assume $k>1$. Then $e_{k}=1$ is the identity element of $S$. Clearly 1 covers $e=e_{k-1}$. By the induction hypothesis, it suffices to show that $\operatorname{dim} \cdot e S=n-1$. Let $\operatorname{dim} \cdot e S=m$ Then $m<n$. Consider the map $\phi: S \rightarrow e S$ given by $\phi(x)=e x$. 
By Lemma 1.9, 1 lies in a unique irreducible component $T$ of $\phi^{-1}(e)$ and $T^{2} \subseteq T$. By [9, Theorem 4.1], $\operatorname{dim} \cdot T \geqslant n-m$. By Lemma 3.6, $e \in T$. Hence $e$ is the zero of $T$ and $T \backslash\{e\}$ is a group. By Lemma 3.11, $\operatorname{dim} \cdot T=1$. Hence $m=n-1$, proving the theorem.

THEOREM 3.18. Let $S$ be connected regular semigroup such that $\operatorname{dim} \cdot S<2$. Then $S$ is a Clifford semigroup and $|\mathcal{Q}(S)| \leqslant 4$.

Proof. By [11, Theorems 2.11, 2.13, 2.15], $S$ is a Clifford semigroup. By Theorem 3.16 , we are reduced to the case when $S$ is a connected $d$-semigroup with zero. We must show that $|E(S)| \leqslant 4$. Suppose $|E(S)| \geqslant 5$. Let $S$ be a closed subsemigroup of $\left(K^{n}, \cdot\right)$ for some $n \in \mathbf{Z}^{+}$. Of all such pairs $(S, n)$ choose one with $n$ minimal. If $n \leqslant 2$, then $|E(S)| \leqslant 4$, a contradiction. So $n \geqslant 3$. Let $e$ denote the identity of $S$. By the minimality of $n, e=(1,1, \ldots, 1)$. Let $G=\left\{\left(a_{1}, \ldots, a_{n}\right) \mid\left(a_{1}, \ldots, a_{n}\right) \in S\right.$, $\left.a_{i} \neq 0, i=1, \ldots, n\right\}$. Then $G$ is the group of units of $S$. By [11, Theorem 2.13], $\operatorname{dim} \cdot S=2$. By Lemma $1.1, \tilde{G}$ is connected and $\operatorname{dim} \cdot \tilde{G}=2$. Let $H=$ $\{(a, b, 1 / a b) \mid a, b \in K, a \neq 0, b \neq 0\}$ under multiplication. By [9, Theorem 16.2], $\tilde{G}$ is ${ }^{*}$-isomorphic to $H$. Hence we have a ${ }^{*}$-homomorphism $\psi: H \rightarrow S$ such that $\psi(H)=G$. Since $S \backslash G$ is closed and $S$ is connected, $\overline{\psi(H)}=S$. Let $\psi=$ $\left(\psi_{1}, \ldots, \psi_{n}\right)$, where $\psi_{i}: H \rightarrow K \backslash\{0\}$. Then each $\psi_{i}$ is a rational character of $\boldsymbol{H}$ and hence [9, p. 102], $\psi_{i}(a, b, 1 /(a b))=a^{\alpha_{i}} b^{\beta_{i}}$ for some $\alpha_{i}, \beta_{i} \in \mathbf{Z}$. Hence $G=$ $\left\{\left(a^{\alpha_{1}} b^{\beta_{1}}, \ldots, a^{\alpha_{n}} b^{\beta_{n}}\right) \mid a, b \in K, a, b \neq 0\right\}$. Let $\mathcal{G}$ denote the free abelian group in variables $X, Y$. Let $u_{i}=X^{\alpha_{i}} Y^{\beta_{i}}, i=1, \ldots, n$. Then $\left(\alpha_{1}, \beta_{1}\right),\left(\alpha_{2}, \beta_{2}\right),\left(\alpha_{3}, \beta_{3}\right)$ are linearly dependent over $\mathbf{Q}$. Hence there exist $\varepsilon_{1}, \varepsilon_{2}, \varepsilon_{3} \in \mathbf{Z}$ such such $\left|\varepsilon_{1}\right|+\left|\varepsilon_{2}\right|+$ $\left|\varepsilon_{3}\right|>0$ and $u_{1}^{e_{1}} u_{2}^{\varepsilon_{2}} u_{3}^{e_{3}}=1$. Relabeling the $u_{i}$ 's if necessary, we can assume that there exist $r_{1}, r_{2}, r_{3} \in Z^{+}$such that one of the following holds: (1) $u_{1}^{r_{1}}=u_{2}^{r_{2}} u_{3}^{r_{3}}$, (2) $u_{1}=1$, (3) $u_{1}^{r_{1}} u_{2}^{r_{2}}=1$, (4) $u_{1}^{r_{1}} u_{2}^{r_{2}} u_{3}^{r_{3}}=1$, (5) $u_{1}^{r_{1}}=u_{2}^{r_{2}}$. If (1) holds then $x_{1}^{r_{1}}=x_{2}^{r_{2}} x_{3}^{r_{3}}$ for all $\left(x_{1}, \ldots, x_{n}\right) \in G$. Since $\bar{G}=S$, the same holds for all $\left(x_{1}, \ldots, x_{n}\right) \in S$. Similarly if (3) holds then $x_{1}^{r_{1}} x_{2}^{r_{2}}=1$ for all $\left(x_{1}, \ldots, x_{n}\right) \in S$. Define $\phi: S \rightarrow K^{n-1}$ as $\phi\left(a_{1}, \ldots, a_{n}\right)=\left(a_{2}, \ldots, a_{n}\right)$ and let $S_{1}=\overline{\phi(S)}$. Then by [11, Example 3.1], $S_{1}$ is a closed connected subsemigroup of $\left(K^{n-1}, \cdot\right)$ and $\operatorname{dim} \cdot S_{1} \leqslant \operatorname{dim} \cdot S=2$. By our induction hypothesis, $\left|E\left(S_{1}\right)\right| \leqslant 4$. Clearly $\phi(E(S)) \subseteq E\left(S_{1}\right)$. Let $e=\left(e_{1}, \ldots, e_{n}\right)$ $\in E(S)$. If (1) holds then $e_{1}=e_{2} e_{3}$. If (2), (3) or (4) holds then $e_{1}=1$. If (5) holds then $e_{1}=e_{2}$. Thus in each of the cases, $|E(S)| \leqslant\left|E\left(S_{1}\right)\right| \leqslant 4$, a contradiction. This proves the theorem.

By Theorems 3.16 and 3.18 we have

COROLlaRY 3.19. Let $S$ be a connected regular semigroup such that $\operatorname{dim} \cdot S=3$ and $S$ is not a d-semigroup with zero. Then $|\mathcal{U}(S)| \leqslant 4$.

TheOREM 3.20. Let $S$ be a connected Clifford semigroup. Then $U(S)$ has the following properties.

(1) $\mathcal{Q}(S)$ is a finite, relatively complemented lattice.

(2) All maximal chains in $\mathcal{U}(S)$ have the same number of elements.

(3) Suppose $\alpha, \beta, \gamma \in \mathcal{U}(S)$ such that $\alpha$ covers $\beta$ and $\beta$ covers $\gamma$. Let $A=\{\delta \mid \delta \in$ थ $(S), \alpha>\delta>\gamma\}$. Then $|A|=2$. 
Proof. By Theorem 3.16 we can assume that $S$ is a $d$-semigroup with zero. (2) then follows from Theorem 3.17. Next we prove (1). Since $U(S) \cong E(S)$ we must show that $E(S)=E$ is relatively complemented. Let $e, f \in E, f<e$. We need to show that $[f, e]$ is complemented. We can assume that $e=1$ is the identity element of $S$ (otherwise we work with $e S$ ). Let $g \in E, g>f, g \neq 1$. We must show that there exists $h \in E$ such that $g h=f$ and $g \vee h=1$. Since $g f=f$, there exists a maximal $h \in E$ such that $g h=f$. Let $e_{1}=g \vee h$. We claim that $e_{1}=1$. Suppose not. Then by Lemma 3.8, there exists $h_{1} \in E$ such that $h_{1}>h$ and $e_{1} h_{1}=h$. Then $g h_{1}=\left(g e_{1}\right) h_{1}=g h=f$. This contradicts the maximality of $h$. Hence $E$ is relatively complemented.

Finally we prove (3). So let $e, f, g \in E$ such that $e$ covers $f$ and $f$ covers $g$. Let $B=\{h \mid h \in E, e>h>g\}$. We must show that $|B|=2$. We can assume that $e=1$ is the identity element of $S$ (otherwise we work with $e S$ ). By Lemma 3.9, there exists a connected closed subsemigroup $T$ of $S$ such that $1, g \in T, g$ is the zero of $T$, and $B \subseteq T$. By Lemma 3.1 and Corollary 3.15, $T$ is a $d$-semigroup. Clearly $\{g<f<1\}$ is a maximal chain in $T$. By Theorem 3.17, dim $\cdot T=2$. Since $g, f$, $1 \in E(T),|E(T)| \geqslant 3$. If $|E(T)|=3$, then $E(T)$ is not relatively complemented, contradicting the above. So by Theorem $3.18,|E(T)|=4$ and $|B|=2$. This proves the theorem.

THEOREM 3.21. Let $S$ be a connected Clifford semigroup with zero 0 such that $\operatorname{dim} \cdot S=3$. Let $A=\{e \mid e \in E(S)$, e covers 0$\}$. Then $|A| \geqslant 3$ and $|E(S)|=2|A|+$ 2.

Proof. By Corollary 3.15, $S$ is a $d$-semigroup. Let $B=\{f \mid f \in E(S), f$ covers $e$ for some $e \in A\}$. Let 1 denote the identity element of $S$. By Theorem 3.17, $E(S)=A \cup B \cup\{1,0\}$ and 1 covers $f$ for all $f \in B$. By Theorem 3.20 (3), each $f \in B$ covers exactly two elements of $A$ and each $e \in A$ is covered by exactly two elements of $B$. Thus by Lemma $1.11,|A|=|B|$. Hence $|E(S)|=2|A|+2$. Suppose $|A|=1$. Then $E(S)$ is linearly ordered, $|E(S)|=4$, contradicting Theorem 3.20 (1). Next assume $|A|=2=|B|$. Let $A=\left\{e_{1}, e_{2}\right\}, B=\left\{f_{1}, f_{2}\right\}$. Then $f_{1}>e_{1}, f_{2}>e_{1}$. So $e_{1}=f_{1} f_{2}$. Similarly $e_{2}=f_{1} f_{2}$ and $e_{1}=e_{2}$, a contradiction. Hence $|A|>3$, proving the theorem.

THEOREM 3.22. Let $n \in \mathbf{Z}^{+}, n \geqslant 3$. Then there exists a closed connected subsemigroup $S$ of $\left(K^{n}, \cdot\right)$ such that $S$ has a zero, $\operatorname{dim} \cdot S=3$ and $|E(S)|=2 n+2$.

Proof. By Lemma 1.8, there exist $u_{1}, \ldots, u_{n} \in \mathscr{F}\left(X_{1}, X_{2}, X_{3}\right)$ such that $u_{i}^{t}$ is not in the semigroup generated by the remaining $u_{j}$ 's for any $i \in\{1, \ldots, n\}, t \in \mathbf{Z}^{+}$. Define $\phi:\left(K^{3}, \cdot\right) \rightarrow\left(K^{n}, \cdot\right)$ as $\phi(a, b, c)=\left(u_{1}(a, b, c), \ldots, u_{n}(a, b, c)\right)$. Then $\phi$ is a *-homomorphism. Let $W=\phi\left(K^{3}\right), S=\bar{W}$. By [11, Example 3.1], $S$ is a closed connected subsemigroup of $\left(K^{n}, \cdot\right)$ and $\operatorname{dim} \cdot S<3$. Clearly $0=(0, \ldots, 0), 1=$ $(1, \ldots, 1) \in W \subseteq S$. Let $e_{i}=(0, \ldots, 1, \ldots, 0)$ be the element of $K^{n}$ with 1 in the $i$ th component and 0 everywhere else. We claim that $e_{i} \in S, i=1, \ldots, n$. Suppose $e_{j} \notin S$ for some $j \in\{1, \ldots, n\}$. Then there exists $f=f\left(Y_{1}, \ldots, Y_{n}\right) \in$ $K\left[Y_{1}, \ldots, Y_{n}\right]$ such that $f\left(a_{1}, \ldots, a_{n}\right)=0$ for all $\left(a_{1}, \ldots, a_{n}\right) \in W$ and $f\left(e_{j}\right) \neq 0$. 
Since $0 \in W, f$ has zero constant term. Now $f=\sum_{i=1}^{n} \alpha_{i} f_{i}$ for some $f_{1}, \ldots, f_{n} \in$ $\mathscr{F}\left(Y_{1}, \ldots, Y_{n}\right), \alpha_{1}, \ldots, \alpha_{n} \in K \backslash\{0\}$ such that $f_{i} \neq f_{k}$ for any $i \neq k$. Since $f\left(e_{j}\right) \neq$ $0, f_{p}=X_{j}^{r}$ for some $p \in\{1, \ldots, n\}, r \in \mathbf{Z}^{+}$. Since $f\left(a_{1}, \ldots, a_{n}\right)=0$ for all $\left(a_{1}, \ldots, a_{n}\right) \in W, f\left(u_{1}, \ldots, u_{n}\right)=0$ in $K\left[X_{1}, X_{2}, X_{3}\right]$. Let $w_{i}=f_{i}\left(u_{1}, \ldots, u_{n}\right) \in$ $\mathscr{F}\left(X_{1}, X_{2}, X_{3}\right)$. Then in $K\left[X_{1}, X_{2}, X_{3}\right], \sum_{i=1}^{n} \alpha_{i} w_{i}=0$. Since $\alpha_{i} \neq 0$ for all $i, w_{q}=w_{p}$ for some $q \in\{1, \ldots, n\}$ such that $p \neq q$. Now $f_{q}=Y_{j}^{s} g$ for some $g \in$ $\mathscr{F}\left(Y_{1}, \ldots, Y_{i-1}, Y_{i+1}, \ldots, Y_{n}\right)^{1}, s \in \mathbf{Z}, s \geqslant 0$. Since $w_{p}=w_{q}$ and $f_{p} \neq f_{q}, g \neq 1$ and $s<r$. Let $v=g\left(u_{1}, \ldots, u_{n}\right) \in \mathscr{F}\left(X_{1}, X_{2}, X_{3}\right)$. Then $v \in\left\langle u_{1}, \ldots, u_{j-1}\right.$, $\left.u_{j+1}, \ldots, u_{n}\right\rangle$ and $u_{j}^{r}=u_{j}^{s} v$. So $r-s \in \mathbf{Z}^{+}$and $u_{j}^{r-s}=v \in\left\langle u_{1}, \ldots, u_{j-1}\right.$, $\left.u_{j+1}, \ldots, u_{n}\right\rangle$, a contradiction. Hence $e_{i} \in S$ for $i=1, \ldots, n$. So $|E(S)|>5$ and by Theorem 3.18, $\operatorname{dim} \cdot S=3$. Clearly $e_{1}, \ldots, e_{n}$ cover 0 . Let $e \in E(S)$ such that $e$ covers 0 . Then $e \geqslant e_{i}$ for some $i$ and so $e=e_{i}$. By Theorem 3.21, $|E(S)|=2 n+2$. This proves the theorem.

Lemma 3.23. Suppose ch $K=0$ and let $S$ be a commutative connected Clifford semigroup, $e, f \in E(S), e>f$. Let $G, H$ be the $\mathcal{H}$-classes of $e$ and $f$ respectively. Define $\delta: \tilde{G} \rightarrow \tilde{H}$ as $\delta\left(a, a^{-1}\right)=\left(a f, a^{-1} f\right)$. Then for any $x \in \tilde{G}_{u}, \delta(x)=(f, f)$ implies that $x=(e, e)$.

Proof. There exist $e_{1}, \ldots, e_{n} \in E(S)$ such that $e=e_{1}>e_{2}>\cdots>e_{n}=f$ and $e_{i}$ covers $e_{i+1}, i=1, \ldots, n-1$. Let $G^{(i)}$ be the $\mathcal{H}$-class of $e_{i}, i=1, \ldots, n$. Consider the *-homomorphisms $\delta_{i}: \tilde{G}^{(i-1)} \rightarrow \tilde{G}^{(i)}$ given by $\delta_{i}\left(a, a^{-1}\right)=\left(a e_{i}, a^{-1} e_{i}\right)$, $i=2, \ldots, n$. Then $\delta=\delta_{n} \circ \ldots \circ \delta_{2}$. Let $W_{i}=\tilde{G}_{u}^{(i)}, i=1, \ldots, n$. By [9, Theorem 15.3], $\delta_{i}\left(W_{i-1}\right) \subseteq W_{i}, i=2, \ldots, n$. Let $\lambda_{i}$ be the restriction of $\delta_{i}$ to $W_{i-1}$, $i=2, \ldots, n$. It suffices to show that each $\lambda_{i}$ is injective. Thus we are reduced to the case when $e$ covers $f$. We can assume that $e=1$ is the identity element of $S$ (otherwise we work with $e S$ ). Let $\theta: \tilde{G} \rightarrow S$ be given by $\theta\left(a, a^{-1}\right)=a$. Let $T=\{a \mid a \in S$, af $=f\}$. By Lemma 1.9, 1 lies in a unique irreducible component $T_{1}$ of $T, T_{1}^{2} \subseteq T_{1}$. By Lemma 3.6, $f \in T_{1}$. So $V=T_{1} \backslash\{f\} \subseteq G$ and by Lemma 3.11, $\tilde{V}_{s}=\tilde{V}$. Let $N$ denote the kernel of $\delta$. Then $\theta(N) \subseteq T$. By [9, Theorem 15.5], $\tilde{G}_{u}$ is a closed subgroup of $\tilde{G}$. Let $N_{1}$ be the irreducible component of $(1,1)$ in $N \cap \tilde{G}_{\boldsymbol{u}}$. Since $1 \in \theta\left(N_{1}\right) \subseteq T$, we see that $\theta\left(N_{1}\right) \subseteq V$. So $N_{1} \subseteq \tilde{V}=\tilde{V}_{s}$. It follows that $N_{1}=\{(1,1)\}$. By [9, Proposition 7.3], $N \cap \tilde{G}_{u}$ is a finite group. Let $x \in N \cap \tilde{G}_{u}$. Then $x$ is unipotent and has finite order. Since $\mathrm{ch} \cdot K=0$ it follows $[9, \mathrm{p} .101$, Exercise 5] that $x=(1,1)$. This proves the lemma.

Theorem 3.24. Suppose ch $\cdot K=0$ and let $S$ be a commutative connected Clifford semigroup. Then there exist a connected abelian unipotent group $W$ and a closed connected subsemigroup $D$ of $S$ such that $D$ is a d-semigroup and $S$ is isomorphic to $W \times D$. Moreover the corresponding isomorphism $\phi: W \times D$ onto $S$ is a morphism of varieties.

Proof. Let 1 denote the identity element of $S$ and $G$ the group of units of $S$. By Theorem 3.16, there exists a closed connected subsemigroup $S_{1}$ of $S$ such that $S_{1}$ is a $d$-semigroup with zero and $E(S) \subseteq S_{1}$. Let $H=S_{1} \cap G$. Then $H$ is the group of units of $S_{1}$. By Lemma 3.13 and Theorem $3.14, \tilde{H}_{s}=\tilde{H}$. Since $\tilde{H} \subseteq \tilde{G}$ we see that 
$\tilde{H} \subseteq \tilde{G}_{s}$. Let $\theta: \tilde{G} \rightarrow S$ be given by $\theta\left(a, a^{-1}\right)=a$ and set $D=\bar{\theta}\left(\tilde{G}_{s}\right)$. Then $H \subseteq D$ and so $S_{1}=\bar{H} \subseteq D$. By [11, Example 3.1], $D$ is a closed subsemigroup of $S$. By Lemma 1.1 and [9, Theorem 15.5], $\tilde{G}_{s}$ is a closed connected subgroup of $\tilde{G}$. Hence $D$ is connected. Let $V=D \cap G$. Then $V$ is the group of units of $D$. By Lemma $1.1, V=\theta\left(\tilde{G}_{s}\right)$. Hence $\tilde{V}=\tilde{G}_{s}$. Thus $\tilde{V}_{s}=\tilde{V}$. Let $M$ denote the kernel of $D$. By Theorem 3.4 (3), $\tilde{M}$ is a ${ }^{*}$-homomorphic image $\tilde{V}$. Hence [9, Theorem 15.3], $\tilde{M}_{s}=\tilde{M}$. Thus $M_{s}=M$ and by [9, Proposition 15.4], $M$ is a $d$-group. By Theorem 3.14, $D$ is a $d$-semigroup. Let $W=\tilde{G}_{u}$ and define $\phi: W \times D \rightarrow S$ as $\phi(x, a)=$ $\theta(x) a . \phi$ is clearly a ${ }^{*}$-homomorphism. Let $a \in S$. Then $a \mathcal{F} e$ for some $e \in E(S)$. Let $F=H_{e}$. Consider the ${ }^{*}$-homomorphism $\delta: \tilde{G} \rightarrow \tilde{F}$ given by $\delta\left(b, b^{-1}\right)=$ $\left(e b, e b^{-1}\right)$. By Theorem $3.4(3), \delta$ is surjective. So there exists $a_{1} \in G$ such that $e a_{1}=a$. Thus $x=\left(a_{1}, a_{1}^{-1}\right) \in \tilde{G}$. So [9, Theorem 15.3] there exist $y \in \tilde{G}_{s}, z \in \tilde{G}_{u}$ $=W$ such that $y z=x$. Let $y=\left(b, b^{-1}\right), z=\left(c, c^{-1}\right)$. Then $b c=a_{1}, b=\theta(y) \in$ $\theta\left(\tilde{G}_{s}\right) \subseteq D$. Now $e \in S_{1} \subseteq D$. So $e b \in D$. Now $\phi(z, e b)=c e b=e a_{1}=a$. Hence $\phi$ is surjective.

We are left with the task of showing that $\phi$ is injective. Suppose $\phi(x, c)=$ $\phi(y, d)$ for some $x, y \in W, c, d \in D$. Let $x=\left(a, a^{-1}\right), y=\left(b, b^{-1}\right)$. Then $a c=b d$. Since $a, b \in G, c \mathcal{H} a c=b d \mathcal{H} d$. Let $c \mathcal{H}\left(e, e \in E(S)\right.$. Set $F=H_{e}$. Since $a c=b d$, $a b^{-1} e=c^{-1} d=w \in F \cap D=Y$. Since $\tilde{M}_{s}=\tilde{M}$ we see by Lemma 3.13 that $\left(w, w^{-1}\right) \in \tilde{Y}=\tilde{Y}_{s} \subseteq \tilde{F}_{s}$. Consider the ${ }^{*}$-homomorphism $\delta: \tilde{G} \rightarrow \tilde{F}$ given by $\delta\left(q, q^{-1}\right)=\left(e q, e q^{-1}\right)$. Since $\left(a b^{-1}, b a^{-1}\right) \in \tilde{G}_{u}$ and $\delta\left(a b^{-1}, b a^{-1}\right)=\left(w, w^{-1}\right)$ we see by $\left[9\right.$, Theorem 15.3] that $\left(w, w^{-1}\right) \in \tilde{F}_{u}$. Hence $\left(w, w^{-1}\right)=(e, e)$. By Lemma 3.23, $a b^{-1}=1$. So $a=b$ and $c=d$. Hence $(x, c)=(y, d)$ and $\phi$ is injective. This proves the theorem.

REMARK 3.25. It is not known to us whether the map $\phi$ in Theorem 3.24 can be chosen to be a ${ }^{*}$-isomorphism, i.e. we do not know whether $\phi$ can be chosen so that $\phi^{-1}$ is also a morphism of varieties.

\section{Examples and problems.}

EXAMPLE 4.1. Let $S=K^{4}$ with multiplication

$$
(a, b, c, d)\left(a_{1}, b_{1}, c_{1}, d_{1}\right)=\left(a a_{1}, a b_{1}+b d_{1}, d c_{1}+c a_{1}, d d_{1}\right) .
$$

Then $S$ is a connected semigroup with identity element $(1,0,0,1)$. Let $T=$ $\{(a, b, c, 0) \mid a, b, c \in K\}$. Then $T$ is a closed connected ideal of $S$. Let $e=$ $(1,0,0,0), \operatorname{Ses}=T e T=T^{2}=\left\{\left(a a_{1}, a b_{1}, c a_{1}, 0\right) \mid a, a_{1}, b_{1}, c \in K\right\}$ is not closed and $\overline{S e S}=T$. This answers in the negative [11, Problem 3.7].

EXAMPLE 4.2. Let $S=K^{2}$ with multiplication $(a, b)\left(a_{1}, b_{1}\right)=\left(a a_{1}, a b_{1}\right)$. Then $S$ is a connected semigroup in which every ideal is closed. However $S$ is not regular. The closed subsemigroup $\{(0,0),(0,1)\}$ of $S$ shows that Theorem 2.6 is not true without the assumption that the semigroup is connected.

EXAMPLE 4.3. Let $n \in \mathbf{Z}^{+}, n \geqslant 2, S=\pi_{n}(K)$ under multiplication. Then $S$ is an example of a semigroup satisfying the hypothesis of Theorems $2.10,2.11,2.12$ and 2.13. This example also shows that Theorem 3.7 and (1), (3) of Theorem 3.20 are not true for connected regular semigroups. 
EXAMPLE 4.4. Let $S=K^{4}$ with multiplication

$$
\left(a_{1}, a_{2}, a_{3}, a_{4}\right)\left(b_{1}, b_{2}, b_{3}, b_{4}\right)=\left(a_{4} b_{3}+a_{1}+b_{1}, a_{2} b_{2}, a_{2} b_{3}+a_{3}, a_{4} b_{2}+b_{4}\right) \text {. }
$$

Then $S$ is a connected Clifford semigroup with identity element $(0,1,0,0)$. Let $G=\{(a, b, c, d) \mid a, b, c, d \in K, b \neq 0\}$. Then $G$ is the group of units of $S$. Let $P=S \backslash G$. Then $P$ is the completely simple kernel of $S$ such that $E(P)$ is not a semigroup. This example shows that the converse of the last statement of Theorem 2.13 (2) is not true.

Problem 4.5. Let $S$ be a connected regular semigroup. Does there exist a commutative subsemigroup $\Lambda$ of $E(S)$ such that $|J \cap \Lambda|=1$ for all $J \in \mathcal{U}(S)$ and $J_{e} \wedge J_{f}=J_{e f}$ for all $e, f \in \Lambda$ ? Theorem 3.16 shows that this is true for connected Clifford semigroups.

EXAMPLE 4.6. Let $S$ be the following band.

\begin{tabular}{c|ccccc} 
& 1 & $e_{1}$ & $f_{1}$ & $e$ & $f$ \\
\hline 1 & 1 & $e_{1}$ & $f_{1}$ & $e$ & $f$ \\
$e_{1}$ & $e_{1}$ & $e_{1}$ & $f$ & $e$ & $f$ \\
$f_{1}$ & $f_{1}$ & $e$ & $f_{1}$ & $e$ & $f$ \\
$e$ & $e$ & $e$ & $f$ & $e$ & $f$ \\
$f$ & $f$ & $e$ & $f$ & $e$ & $f$
\end{tabular}

Then $\mathscr{U}(S)$ is a lattice, $|\mathcal{U}(S)|=4$. However no subsemilattice of $S$ passes through each $g$-class of $S$. Hence no analogue of Theorem 3.16 holds true for abstract Clifford semigroups.

EXAMPLE 4.7. Let $n \in \mathbf{Z}^{+}, n \geqslant 2$ and let

$$
\begin{aligned}
& S=\left\{\left(x_{1}, y_{1}, \ldots, x_{n}, y_{n}\right) \mid x_{1}, \ldots, x_{n}, y_{1}, \ldots, y_{n} \in K\right. \\
& \left.\quad \text { and } x_{i} y_{j}=x_{j} y_{i}, i, j=1, \ldots, n\right\} \subseteq K^{2 n} .
\end{aligned}
$$

Then $S$ is a closed subsemigroup of $\left(K^{2 n}, \cdot\right)$. Define $\phi: K^{n+2} \rightarrow S$ as $\phi\left(a_{1}, \ldots, a_{n}, u, v\right)=\left(a_{1} u, a_{1} v, \ldots, a_{n} u, a_{n} v\right)$. Then $\phi\left(K^{n+2}\right)=S$. Hence $S$ is a connected $d$-semigroup with zero. Note that $\operatorname{dim} \cdot S=n+1$ and $|E(S)|=|U(S)|$ $=3\left(2^{n}\right)-2$. Let $n=2$. Then $\operatorname{dim} \cdot S=3,|E(S)|=10$. It is easily verified that $E(S)$ is not a semimodular or a lower semimodular lattice (see [4]).

EXAMPLE 4.8. Let $S=\left\{(a, b, c) \mid a, b, c \in K, a^{2} b=c^{2}\right\}$ considered as a closed subsemigroup of $\left(K^{2}, \cdot\right)$. Then $S$ is a connected $d$-semigroup with zero and $\operatorname{dim} \cdot S=2$. Let $f=(0,1,0)$. Then $f \in E(S)$. The semigroup

$$
T=\{x \mid x \in S, x f=f\}=\left\{(a, 1, c) \mid a, c \in K, a^{2}=c^{2}\right\}
$$

is clearly not connected. Thus Lemma 3.6 cannot be improved further.

EXAMPLE 4.9. Let $m \in \mathbf{Z}^{+}$and let $S=\left\{(a, b, c) \mid a, b, c \in K, a^{m} c=b^{m}\right\}$ with multiplication $(a, b, c)\left(a_{1}, b_{1}, c_{1}\right)=\left(a a_{1}, a b_{1}, c_{1}\right)$. Then $S$ is a connected Clifford semigroup with $\operatorname{dim} \cdot S=|\mathcal{Q}(S)|=2$. Let $g=(0,0,1)$. Then

$$
V=\{f \mid f \in E(S), f \geqslant g\}=\left\{(1, \alpha, 1) \mid \alpha \in K, \alpha^{m}=1\right\} \cup\{g\} .
$$

Suppose $\mathrm{ch} \cdot K=0$. Then $|V|=m+1$. Thus the set considered in Theorem 3.7 can be arbitrarily large even when $|\mathcal{Q}(S)|=2$. In particular, $f$ in Theorem 3.4 (1) 
need not be unique. Next replace $K$ by the field of real numbers R. Let $m=2$ and let $f \in(0,0,-1), e=(1,1,1)$. Then $e \mid f, e, f \in E(S)$. However there is no $e_{1} \in$ $E(S)$ such that $e g_{e_{1}}$ and $e_{1} \geqslant e$. Hence Theorem $3.4(1)$ is false if $K$ is replaced by R.

Problem 4.10. Let $S$ be a connected Clifford semigroup. Does there exist $m \in \mathbf{Z}^{+}$such that for all $f \in E(S),|\{e \mid e \in E(S), e \geqslant f\}| \leqslant m$ ?

EXAMPLE 4.11. Let $S$ be a connected regular semigroup. Theorem 3.7 suggests the conjecture that if the set $\{e \mid e \in E(S), e \geqslant f\}$ is finite for all $f \in E(S)$, then $S$ is a Clifford semigroup. Unfortunately this conjecture is not true. Let $S=K^{3}$ with multiplication

$$
(a, b, c)\left(a_{1}, b_{1}, c_{1}\right)=\left(\left(1+b c_{1}\right) a a_{1}, b_{1}, c\right) .
$$

Then $S$ is a connected regular semigroup, $\operatorname{dim} S=3$. Let $J_{1}=\{(a, b, c) \mid a, b, c \in$ $K, a \neq 0\}, J_{2}=\{(0, b, c) \mid b, c \in K\}$. Then $\mathcal{U}(S)=\left\{J_{1}, J_{2}\right\}, J_{1}>J_{2}$. Since $(1,1,-1) \in J_{1}$ and $(1,1,-1)^{2}=(0,1,-1) \in J_{2}, S$ is not a Clifford semigroup. Let $b, c \in K$ and set $f=(0, b, c)$. Then $f \in E\left(J_{2}\right)=J_{2}$. Suppose $e \in E\left(J_{1}\right), e \geqslant f$. Then $e=(a, b, c)$ for some $a \in K, a \neq 0$ and $(1+b c) a^{2}=a$. Hence $b c \neq-1$ and $a=1 /(1+b c)$. Thus for all $f \in E(S),|\{e \mid e \in E(S), e \geqslant f\}|<2$. Also note that there is no $e \in E\left(J_{1}\right)$ such that $e \geqslant(0,1,-1)$. So Theorem 3.4 (1) is not true for connected regular semigroups.

Problem 4.12. Determine all possible lattice structures for $U(S)$ when (1) $S$ is a connected semigroup, (2) $S$ is a connected regular semigroup, and (3) $S$ is a connected Clifford semigroup. Theorem 3.16 reduces the third problem to the study of $E(S)$ where $S$ is a connected $d$-semigroup with zero.

\section{REFERENCES}

1. A. Borel, Linear algebraic groups, Benjamin, New York, 1969.

2. A. H. Clifford, Semigroups admitting relative inverses, Ann. of Math. (2) 42 (1941), 1037-1049.

3. A. H. Clifford and G. B. Preston, The algebraic theory of semigroups, Math. Surveys, no. 1, Amer. Math. Soc., Providence, R. I., 1961.

4. P. Crawley and R. P. Dilworth, Algebraic theory of lattices, Prentice-Hall, Englewood Cliffs, N. J., 1973.

5. M. Demazure and P. Gabriel, Groupes algébriques. I, Géométrie algébrique, généralités, groupes commutatifs, North-Holland, Amsterdam, 1970.

6. M. P. Drazin, Natural structures on rings and semigroups with involution (to appear).

7. T. E. Hall, The partially ordered set of all J-classes of a finite semigroup, Semigroup Forum 6 (1973), 263-264.

8. K. Hoffman and R. Kunze, Linear algebra, Prentice-Hall, Englewood Cliffs, N. J., 1971.

9. J. E. Humphreys, Linear algebraic groups, Springer-Verlag, Berlin and New York, 1975.

10. W. D. Munn, Pseudo-inverses in semigroups, Proc. Cambridge Philos. Soc. 57 (1961), 247-250.

11. M. S. Putcha, On linear algebraic semigroups, Trans. Amer. Math. Soc. 259 (1980), 457-469.

12. J. Rhodes, Problems 23-28, Semigroup Forum 5 (1972), 92-94.

13. I. R. Shafarevich, Basic algebraic geometry, Springer-Verlag, Berlin and New York, 1974.

14. T. Tamura, The theory of construction of finite semigroups, Osaka Math. J. 8 (1956), 243-261.

Department of Mathematics, North Carolina State University, Raleigh, North Carolina 27650 